\title{
Optimization of proton therapy eye-treatment systems toward improved clinical performances
}

\author{
Eustache Gnacadja $\odot,{ }^{1, *}$ Cédric Hernalsteens $\odot,{ }^{1,2, \dagger}$ Stewart Boogert, ${ }^{3}$ Quentin Flandroy, ${ }^{4}$ Carolina Fuentes, ${ }^{4}$ \\ Laurence J. Nevay, ${ }^{3}$ Nicolas Pauly $\odot,{ }^{1}$ Eliott Ramoisiaux, ${ }^{1}$ William Shields $\odot,{ }^{3}$ Robin Tesse, ${ }^{1}$ \\ Raphael Van Roermund, ${ }^{4}$ and Marion Vanwelde $\mathbb{C}^{1}$ \\ ${ }^{1}$ Service de Métrologie Nucléaire, Université libre de Bruxelles, Avenue Franklin Roosevelt 50, 1050 Brussels, Belgium \\ ${ }^{2}$ CERN, European Organization for Nuclear Research, 1211 Geneva 23, Switzerland \\ ${ }^{3}$ John Adams Institute at Royal Holloway, University of London, Egham TW20 OEX, United Kingdom \\ ${ }^{4}$ Ion Beam Applications, Chemin du Cyclotron 3, 1348 Ottignies-Louvain-la-Neuve, Belgium
}

(Received 22 July 2021; accepted 5 January 2022; published 11 February 2022; corrected 25 February 2022)

\begin{abstract}
The treatment protocols of cancerous ocular diseases with proton therapy are well established, and dedicated eye-treatment systems can produce the clinical beam properties that meet the peculiar features required by eye-treatment modalities. However, for general-purpose multiroom systems comprising eye-treatment beamlines and nozzles, the design and commissioning procedures must be optimized to achieve the performances of fully dedicated systems in terms of depth-dose distal falloff, lateral penumbra, and dose rate. This paper presents a realistic start-to-end beam transport and particle-matter interactions model of the ion beam applications Proteus ${ }^{\circledR}$ Plus $(\mathrm{P}+)$ single-scattering eye-treatment room with Beam Delivery SIMulation (BDSIM) using Geant4. The model is used to establish optimization patterns in terms of beam optics to achieve a smaller depth-dose distal falloff than the design baseline while maintaining a nominal dose rate and lateral flatness of the dose deposition profile. An alternative design is proposed to increase the dose rate further by up to a factor 3 , allowing for delivering a complete hypofractionated treatment session under $60 \mathrm{~s}$. It uses a beam-stopping device to complement the existing scattering features of the nozzle. An in-depth study of the system is performed using BDSIM and the numerical simulations are discussed in detail.
\end{abstract}

DOI: 10.1103/PhysRevResearch.4.013114

\section{INTRODUCTION}

Proton therapy is a well-established method for the treatment of ocular melanomas [1]. Ocular melanomas are the most common type of primary intraocular cancers. They originate from eye melanocytes (pigment cells) and are rare cancerous tumors which have an occurrence rate of six cases per million adults in the United States [2]. When located in the uveal tract, this malignant tumor is called an uveal melanoma and can arise in the iris, in the ciliary body, or within the choroid. All of these three melanoma types have the potential to metastasize to other parts of the body, most often to the liver.

Although other treatment options exist, including surgery (either local resection or total enucleation), chemotherapy, brachytherapy, and thermotherapy, proton therapy treatments offer advantages given their ability to spare healthy ocular and intracranial tissues. Ocular cancer treatment with proton beams started in 1972. It makes use of the sharp dose deposition profiles of protons to deliver a high-dose treatment field while sparing healthy tissues and nearby organs

\footnotetext{
*eustache.gnacadja@ulb.be

$\dagger$ cedric.hernalsteens@ulb.be
}

Published by the American Physical Society under the terms of the Creative Commons Attribution 4.0 International license. Further distribution of this work must maintain attribution to the author(s) and the published article's title, journal citation, and DOI. at risk (OARs), such as the optic nerve. Several retrospective studies demonstrate satisfactory clinical outcomes for ocular cancer patients treated with proton therapy. Tae Wan et al. [3] reported on 24 patients, who received 60 to 70 cobalt gray equivalent (CGE) dose prescriptions, administrated in 5 fractions. The patients were followed over several years to estimate typical values for 3 years local progression-free and metastasize-free survival rates. The observed values were respectively $95.7 \%$ and $95.8 \%$, and very low health tissue toxicity was recorded.

Efficient proton therapy treatment can be obtained with an axial (frontal) entrance portal. As the required beam penetration depth is limited to the diameter of the eye (typically $2.5 \mathrm{~cm}$ ), and as no dense, bony tissues are encountered, the maximum required proton range is smaller compared to other treatment types by a factor of 10 or more. Additionally, given the small dimensions of the structures of interest, sparing the OARs requires that the dose deposition profiles have sharp edges. The dose distal falloff (DFO) and lateral penumbra (LP) are the key parameters to characterize the dosimetric profiles. The definition of those quantities is illustrated for a typical eye-treatment field in Fig. 1. The depth at which the normalized dose value reaches $90 \%$ on the distal side of the Bragg peak is the distal range $R_{90}$, as illustrated on the depthdose profile of Fig. 1(left). The distal $R_{80}$ and $R_{20}$ are defined similarly. The distal falloff is the difference between the $20 \%$ and $80 \%$ dose points: $\mathrm{DFO}=\mathrm{R}_{80}-\mathrm{R}_{20}$. The field size (FS) is defined as the distance between the $50 \%$ points $\left(\mathrm{D}_{50}\right)$ of the normalized transverse dose profile, as shown on the lateral 

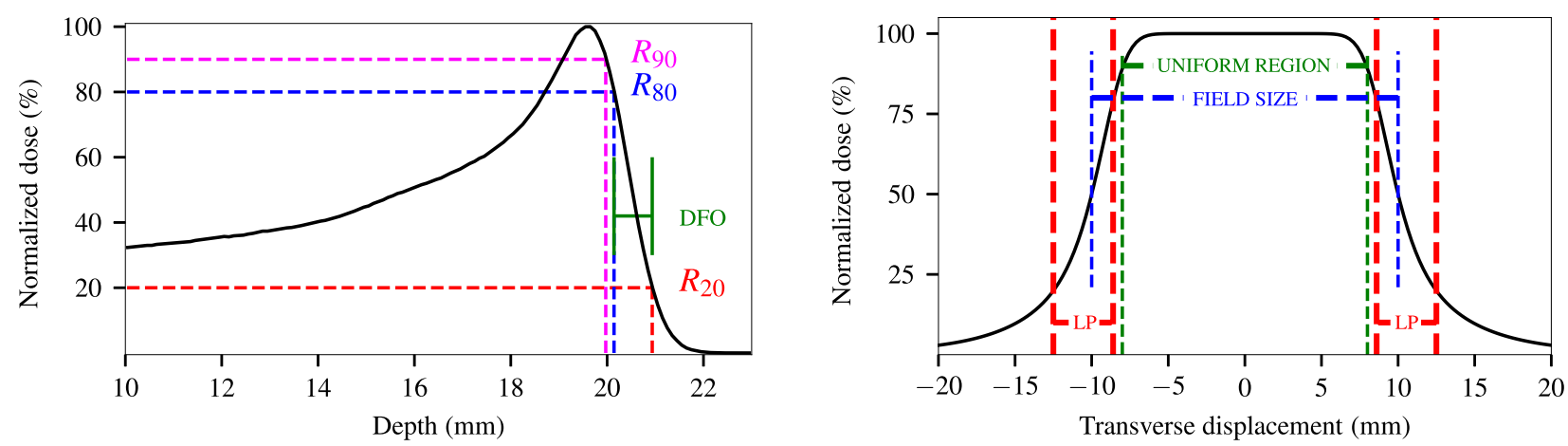

FIG. 1. Definition of the clinical properties for the depth dose deposition pattern (on the left) and lateral dose profile (on the right). The figure on the left shows the distal range values at $90 \%, 80 \%$, and $20 \%$, denoted respectively $\mathrm{R}_{90}, \mathrm{R}_{80}$, and $\mathrm{R}_{20}$. The distal falloff (DFO) is also represented. The figure on the right displays the properties of the lateral dose deposition profile: the field size (FS), the uniform region (UR), and the left and right lateral penumbras (LP).

profile of Fig. 1(right). The lateral penumbra is defined on both sides $\left(\mathrm{LP}_{\mathrm{L}}\right.$ and $\left.\mathrm{LP}_{\mathrm{R}}\right)$ of the transverse profile as the distance between the $20 \%$ and $80 \%$ points. Finally, the uniform region (UR) is defined as $\mathrm{UR}=\mathrm{FS}-2 \times \mathrm{LP}_{\mathrm{L}}-2 \times \mathrm{LP}_{\mathrm{R}}$. Tight constraints are defined for these quantities, despite the variability that exists among treatment centers.

By 2014, proton therapy for ocular tumors treatment was provided by close to 20 centers worldwide [4]. Table I lists established centers with a large number of treated patients for which beam parameters and dosimetric performances are available in the literature. For each center, the characteristics and clinical performances of the beam delivery system are provided for comparison. One can observe that the dedicated systems, featuring a low energy cyclotron, typically achieve both a high dose rate and a very small DFO $(\leqslant 1 \mathrm{~mm})$. Indeed, only a small energy degradation is required in such systems as the beam is extracted from the accelerator close to the required clinical energy leading to a reduced energy spread and range straggling, and to fewer losses. On the other hand, most highenergy systems, ${ }^{1}$ featuring a significant energy degradation, only achieve a DFO in the range $2-6 \mathrm{~mm}$. This is due to the significant energy spread generated during the beam interaction with the energy degrader. Although an energy selection system (ESS) is used for such high energy machines to reduce this energy spread, the required minimum dose rate (typically higher than $10 \mathrm{~Gy} / \mathrm{min}$ ) limits the achievable selection in energy, leading to a DFO that remains higher than the one of low energy systems.

In most centers, the desired beam forming is obtained with single scattering (SS) techniques. SS has the advantage of providing a much smaller LP compared to double scattering (DS) techniques, which are unnecessary for this specific application due to the small field nature of the irradiation (typically lower than $3 \mathrm{~cm}$ ) [16]. Nevertheless, SS has the drawback of leading to significant beam losses. These beam forming losses put an additional limitation on the maximum achievable dose rate,

\footnotetext{
${ }^{1}$ General purpose systems typically provide a clinically achievable range up to $32 \mathrm{~cm}$ using a beam energy at the exit of the accelerator in the range 230 to $250 \mathrm{MeV}$, which is also used for the eye-treatment room in the case of multiroom IBA systems.
}

making it difficult to optimize all the clinical performances of the beamline at the same time.

In this paper, we present a start-to-end optimization procedure for the ion beam applications (IBA) Proteus ${ }^{\circledR}$ Plus $(\mathrm{P}+)$ passive scattering eye-treatment beamline, so-called the "eyeline." The IBA eyeline, whose baseline design uses single scattering techniques, features a beam energy degradation from $230 \mathrm{MeV}$ at the exit of the normal conducting cyclotron to a nominal energy in the $70-105 \mathrm{MeV}$ range. Such an energy degradation, together with the scattering processes, inevitably induces significant beam losses, which puts substantial limitations on the beamline clinical performances. The IBA eyeline was previously installed at the University of Florida-Health Proton Therapy Institute (UFPTI), in Jacksonville. In this previous installation, the nominal energy at the entrance of the nozzle was $105 \mathrm{MeV}$. Such a high energy allows a dose rate up to $30 \mathrm{~Gy} / \mathrm{min}$ with single scattering techniques, but with the drawback of high DFO (3 mm) (see Table I)). The ultimate goal of this paper is to modify the baseline design of the IBA eyeline, to allow a smaller DFO ( $\leqslant 2 \mathrm{~mm})$, while maintaining an acceptable dose rate $(\geqslant 15 \mathrm{~Gy} / \mathrm{min})$.

First, we build a realistic model of the IBA eyeline using beam delivery simulation (BDSIM) [17], a Monte Carlo code that can simulate the propagation of charged particles through 3D beamline models. Our model includes the beam transport system (BTS), the beam forming device (the nozzle), and the main shielding walls of the treatment room.

We develop a numerical approach toward the optimization of the beamline optics and transmission using an in-house particle tracking PYTHON code, which uses beam transfer maps to propagate protons through most common accelerator and beamline magnet types, with the significant advantage of being fast enough to be coupled to optimization routines. The optimization results provide the required beamline configuration to deliver an efficient and clinically acceptable beam at the entrance of the treatment room while maintaining beamline losses at the lowest possible level.

We then present the design of the beam forming nozzle, starting with a detailed optimization of the clinical performances in single scattering mode. We follow this optimization by the study of an alternative design, which combines a first scatterer with a cylindrical beam stopping device to achieve a flat transverse dose profile. We demonstrate that the latter 
TABLE I. Machine and beam parameters for proton therapy eye-treatment centers in clinical operation, ordered by beam energy at room entrance. The beam production system (BPS) energy, the energy used for the eyeline and parameters of dosimetric performances (maximum range in water, distal falloff, and lateral penumbra), are reported. Reconstructed using data from [1] and [4].

\begin{tabular}{llccrc}
\hline \hline & $\begin{array}{c}\text { BPS energy } \\
(\mathrm{MeV})\end{array}$ & $\begin{array}{c}\text { Eyeline energy } \\
(\mathrm{MeV})\end{array}$ & $\begin{array}{c}\text { Maximum range } \\
(\mathrm{mm})\end{array}$ & $\begin{array}{c}\text { Maximum DR } \\
(\mathrm{Gy} / \mathrm{min})\end{array}$ & $\begin{array}{c}\text { DFO } \\
(\mathrm{mm})\end{array}$ \\
\hline IFJ, Kraków [5] & 60.0 & 58.0 & 28.0 & 36.0 & 0 \\
$(\mathrm{~mm})$
\end{tabular}

${ }^{\mathrm{a}}$ The Massaschussets General Hospital (MGH) of the Harvard Medical School in Boston started to treat ocular tumors in 1975, using 162 MeV proton beams delivered by their isochronous research cyclotron. At the beginning of 1994, the NPTC project led to the installation of the IBA isochronous cyclotron, which can deliver a beam up to $230 \mathrm{MeV}$.

design leads to very limited beam scattering losses compared to the SS technique and can significantly increase the maximum achievable dose rate by up to a factor of 3 . Using BDSIM, Monte Carlo simulations were performed to assess the detailed clinical properties of the dose deposition profiles resulting from the two designs. A side-by-side comparison was made to highlight the advantages of the beam stopper solution in terms of dose rate and reduction of the dose at skin.

The BDSIM model of the treatment room is presented in detail in Sec. II, followed by a discussion of the features of the main components in the specific context of eye-treatment beam delivery. Section III is dedicated to the study of the impact of machine parameters on the clinical beam properties at the patient position. This study highlights the advantages of building a numerical model in terms of clinical performance prediction capabilities. In Sec. IV, the workflow toward the beamline optics optimization is discussed in detail. Finally, Secs. V and VI present the detailed design of the beam forming nozzle, using respectively single scattering techniques and the beam stopper.

\section{BDSIM MODEL OF THE IBA EYE- TREATMENT BEAMLINE}

The numerical optimization of the dosimetric properties of particle therapy beamlines requires Monte Carlo tools, to simulate the resulting dose deposition profiles, from which the clinical properties can be accurately computed. BDSIM is a Monte Carlo and particle tracking code which uses the Geant4 [18], ROOT [19], and CLHEP [20] libraries to build geometries for beamline magnets and other accelerator components, together with the associated electromagnetic fields. It allows beam transport through these components, while accounting for the particle-matter interaction processes using the physics models of Geant4. Those physics models have been recently benchmarked against other Monte Carlo simulations tools, especially for low energy proton beam degradation [21]. Highly flexible, BDSIM performs beam tracking based on accelerator tracking routines and Geant4 numerical integrators, and also allows the inclusion of nonstandard machine components, such as an energy degrader, concrete shielding walls, and a dosimetric phantom. Thanks to the physics processes provided by Geant 4 , accurate dose deposition profiles can be simulated, allowing the numerical optimization of the clinical properties of the modeled beamline. Experimental validation of Geant4's nuclear models for proton therapy has been previously carried out, and has shown the ability of this code to accurately simulate the interaction of low energy protons in matter [22]. The capabilities of BDSIM to model proton therapy facilities have already been demonstrated, especially in the context of concrete activation studies [23]. Moreover, its ability to reproduce experimental beam sizes and dose deposition data has been recently demonstrated and discussed in detail [24].

The IBA SS eye-treatment beamline (the "eyeline") is a part of the Proteus ${ }^{\circledR}$ Plus $(\mathrm{P}+)$ system, which is a cyclotron-based, multiroom proton therapy center. In $\mathrm{P}+$, the normal-conducting $\mathrm{C} 230$ cyclotron accelerates protons to $230 \mathrm{MeV}$ toward a short extraction beamline that focuses the beam on a variable energy degrader. The energy required during the course of a treatment session is set by the rotation of the degrader wheel, which is made of different materials, with a variable thickness as a function of the angle. energy selection system (ESS) reduces the energy spread using movable beam limiting slits inserted in a location of large horizontal dispersion. After the ESS a transport system brings the beam to the dual beam transport room (DBTR). The DBTR is the first treatment room of the $\mathrm{P}+$ facility. It features fixed beam treatments using the fixed beam treatment room (FBTR) nozzle, while allowing at the same time eye treatments with the eye-treatment nozzle.

The detailed 3D model of the IBA eye-treatment beamline, programatically built with BDSIM, is shown in Fig. 2. The main components are indicated, together with their locations along the beamline. A simplified model of the $\mathrm{C} 230$ cyclotron is used for its mass and is simulated separately with the 


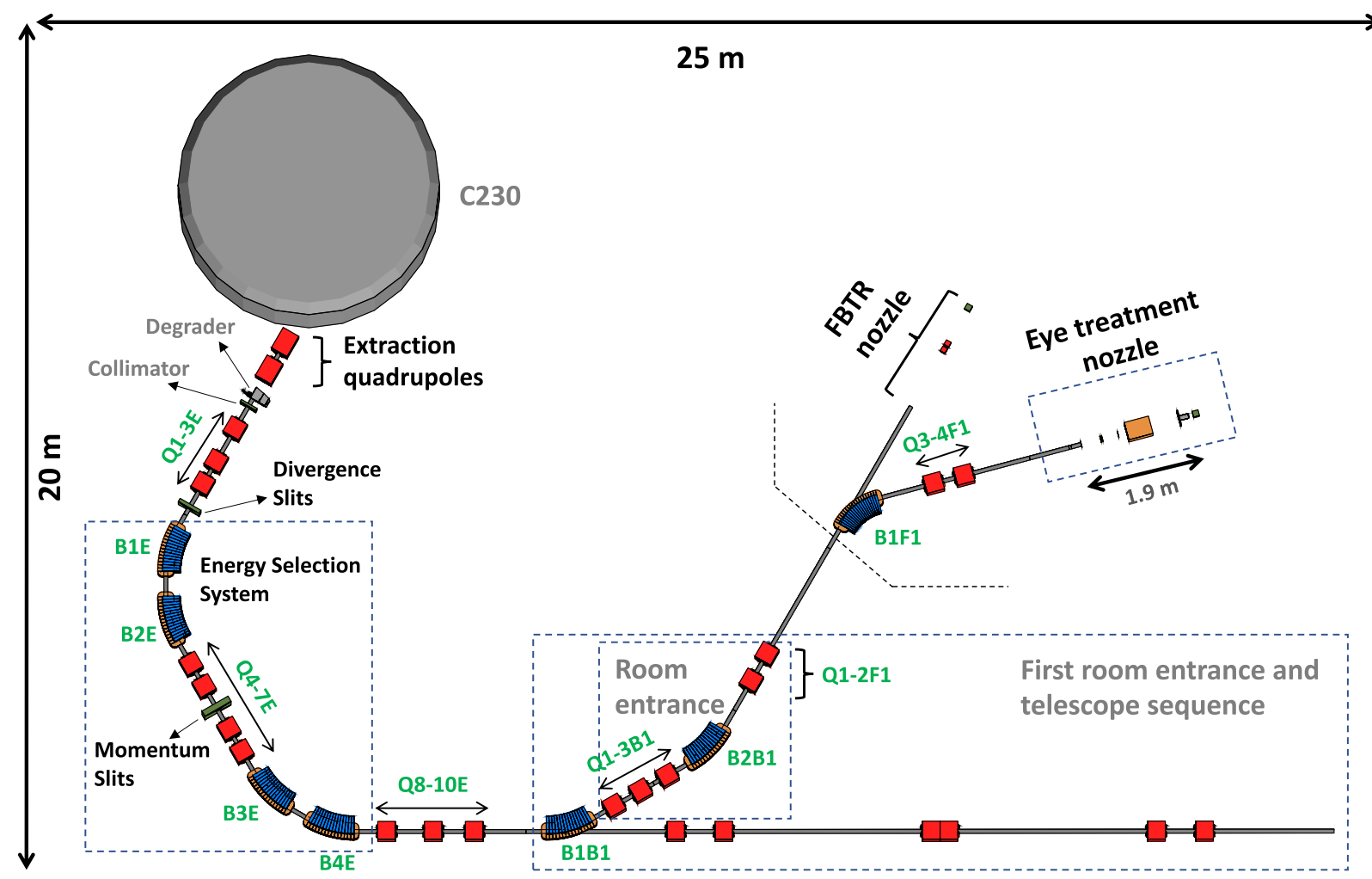

FIG. 2. Dual beam treatment room (DBTR) of the IBA Proteus®Plus system, as modeled with BDSIM. A basic geometric representation of the $\mathrm{C} 230$ cyclotron is visible, followed by the extraction line, which is made of two quadrupoles. The energy selection system (ESS), which consists of the energy degrader, the circular collimator, the first focusing triplet (Q1-3E), and the achromatic system made of four bending magnets (B1-4E) and four quadrupoles (Q4-7E) is also modeled. The main components of the straight fixed line nozzle are also visible. The telescope sequence, which allows the same beam optics at the entrance of each treatment room of the center, is also modeled.

beamline simulation starting at its exit port. The beam transport system (BTS) is made using customized BDSIM-generated geometry and a realistic model of the degrader is included $[23,24]$.
Figure 3 shows schematically the complete model including the $\mathrm{P}+$ shielding walls and the beam forming nozzle. We can observe from left to right the first ionization chamber (IC1), which serves as a beam profile monitor at the nozzle

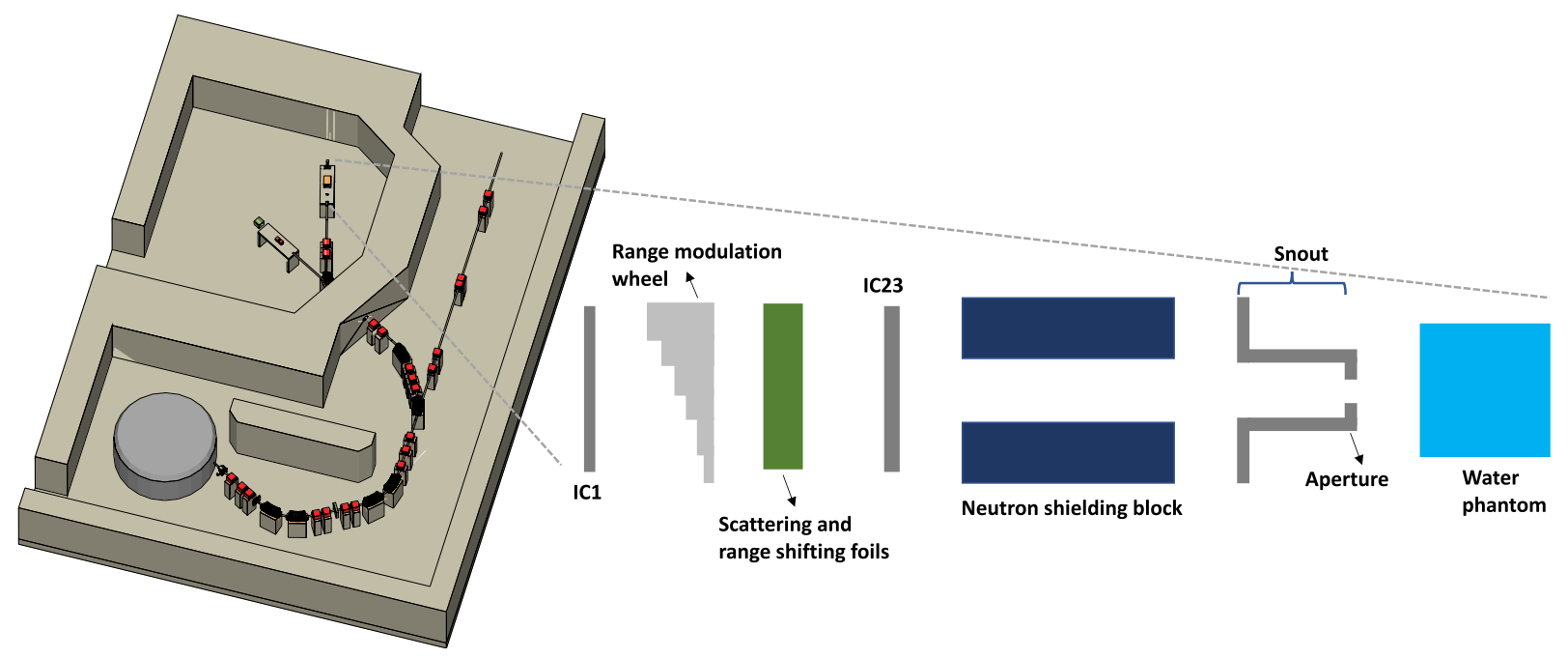

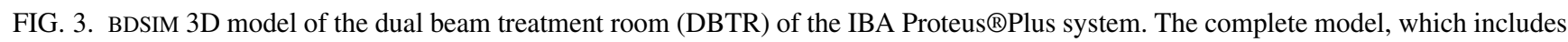
the C230 normal conducting cyclotron, the beam transport system, and the beam forming nozzle, is shown on the left. A detailed schematic representation of the nozzle is shown on the right, including the first ionization chamber (IC1), the scattering and range shifting foils, the second and third ionization chambers (IC23), the neutron shielding hollow block, the snout, the 1.5-cm-radius circular aperture, and the water phantom. 
TABLE II. Twiss parameters of the beam at the entrance of the degrader.

\begin{tabular}{lccc}
\hline \hline Plane & $\alpha$ & $\beta$ & $\epsilon$ \\
\hline $\mathrm{X}$ & 0.0 & $2.0 \mathrm{~m}$ & $1.0 \times 10^{-6} \mathrm{~m}$ \\
$\mathrm{Y}$ & 0.0 & $1.0 \mathrm{~m}$ & $1.5 \times 10^{-7} \mathrm{~m}$ \\
\hline \hline
\end{tabular}

entrance, the range modulation wheel (RMW), used to achieve a uniform depth dose deposition profile, the scattering and range shifting block, which contains the beam scattering (Tantalum, high $Z$ and high density material) and range shifting (Lexan, low $Z$ material) foils that are required to adjust the maximum range and the lateral uniformity of the dose profile, the neutron shielding block, which is a hollow borated polycarbonate cube used to limit the neutron flux generated in the nozzle (mainly by scattering and range shifting processes), the snout, and a $3 \mathrm{~cm}$ inner diameter aperture. The $3 \mathrm{~cm}$ aperture simulated in this work represents the maximum field size to be delivered. Actual patient-specific apertures are typically of smaller radii. A $10 \mathrm{~cm}$ cubic water phantom (WP), placed $7 \mathrm{~cm}$ downstream of the aperture exit, is also shown. Realistic models of the neutron shielding block, the snout, and the aperture are built using pyg4ometry [25], a PYTHON library that allows the conversion and construction of geometries into and from different description formats. The other parts of the nozzle are generic elements of BDSIM. Although the model does not include a realistic geometry of the RMW, its impact on the beam was simulated using variable thickness Lexan slabs.

During treatment, the transverse and depth-dose profiles obtained at the patient position must meet tight clinical requirements imposed on their dosimetric properties. More specifically, the transverse profile LP must typically be below $1.5 \mathrm{~mm}$. The maximum transverse field size (FS) and the penumbra define the uniform region. The dose profile of the uniform region must remain between $98 \%$ and $102 \%$ of the on-axis dose value. The DFO must remain below $2 \mathrm{~mm}$ for all depth dose profiles and an optimal dose rate of at least $15 \mathrm{~Gy} / \mathrm{min}$ must be achieved.

In the context of the numerical approach proposed, the development of a detailed Monte Carlo model of the IBA eyeline is of major interest, as it allows a systematic evaluation of all the clinical performances of the beamline to guide its optimization. Before starting this optimization, the beam characteristics at the entrance of the degrader wheel must be defined. We know that, in a given transverse phase space, a good approximation of the shape of the beam is an ellipse and that the beam characteristics are completely defined by the area, the shape, and the orientation of this ellipse in the two transverse phase spaces.

Before optimizing the beamline, the Twiss parameters of the beam at the entrance of the degrader were adjusted, to fit on-site beam profile monitors' (BPMs) data, for an energy of $70 \mathrm{MeV}$ at the degrader exit. The derived values, which have then been validated at other (higher) energies, are summarized in Table II, for the two transverse phase spaces (X and $\mathrm{Y}$ ). We can observe that the $\alpha$ parameters are equal to 0 in both planes, which means that the beam exhibits a so-called
TABLE III. Clinical requirements imposed on the dosimetric properties of the IBA passive scattering eyeline.

\begin{tabular}{lccccc}
\hline \hline $\begin{array}{l}\mathrm{R}_{\min } \\
(\mathrm{mm})\end{array}$ & $\begin{array}{c}\mathrm{R}_{\max } \\
(\mathrm{mm})\end{array}$ & $\begin{array}{c}\mathrm{DFO} \\
(\mathrm{mm})\end{array}$ & $\begin{array}{c}\text { Flatness }\left(\mathrm{F}_{l}\right) \\
(\%)\end{array}$ & $\begin{array}{c}\mathrm{LP} \\
(\mathrm{mm})\end{array}$ & $\begin{array}{c}\mathrm{DR} \\
(\mathrm{Gy} / \mathrm{min})\end{array}$ \\
\hline 5 & 35 & $<2$ & $<2$ & $<1.5$ & $>15$ \\
\hline \hline
\end{tabular}

"double waist" (i.e., a local minimum size in both transverse planes) at this position. This configuration allows a minimal emittance growth during the beam-matter interactions in the degrader and ultimately limits the losses along the beamline [26].

\section{MACHINE PARAMETERS' IMPACT ON CLINICAL BEAM PARAMETERS}

The clinical requirements on the dosimetric properties of the IBA passive scattering eye-treatment beamline are summarized in Table III.

In Table III, $\mathrm{R}_{\min }$ and $\mathrm{R}_{\max }$ are the minimum and maximum desirable treatment ranges. The dosimetric properties of the beamline must be optimized for all ranges within this interval. The flatness $\mathrm{F}_{l}$ quantifies the uniformity of the dose distribution in the target and applies to both transverse and depth dose profiles as defined by

$$
\mathrm{F}_{l}=100 \times \frac{\mathrm{D}_{\max }-\mathrm{D}_{\min }}{\mathrm{D}_{\max }+\mathrm{D}_{\min }},
$$

where $D_{\min }$ and $D_{\max }$ are respectively the minimum and maximum dose values in the region of interest. For transverse profiles, this applies to the uniform region, while for the depth dose profiles it is defined for the flat top of the spread-out Bragg peaks (SOBPs). The SOBPs are obtained by combining a weighted set of individual Bragg curves, to cover the indepth thickness of the tumor.

We know that the clinical properties result from the beam properties at isocenter (namely the nominal energy $E$, the energy spread $\Delta E / E$, and the transverse distribution of the beam). Those beam properties are in turn defined by several machine parameters of the beamline, more specifically, as follows.

(i) The DFO is defined by the beam nominal energy $E$ (which is set by adjusting the degrader angle) and by the beam energy spread $(\Delta E / E)$ at isocenter. For a given beam nominal energy $E$ at the exit of the degrader, the energy spread $\Delta E / E$ at isocenter is imposed by the ESS (especially the opening of the momentum slits) and by the beam-matter interaction processes within the nozzle. Moreover, due to the range straggling processes in water, the achievable DFO at isocenter also depends on the irradiation range in the patient (the higher the range, the more the straggling processes in water, and the higher the resulting energy spread and DFO).

(ii) The transverse flatness depends on the beam interactions with the scattering and range shifting foils within the nozzle. As the eyeline is a passive scattering system, an efficient (high dose rate) treatment is only achievable by maintaining beam losses within the nozzle at the lowest possible 

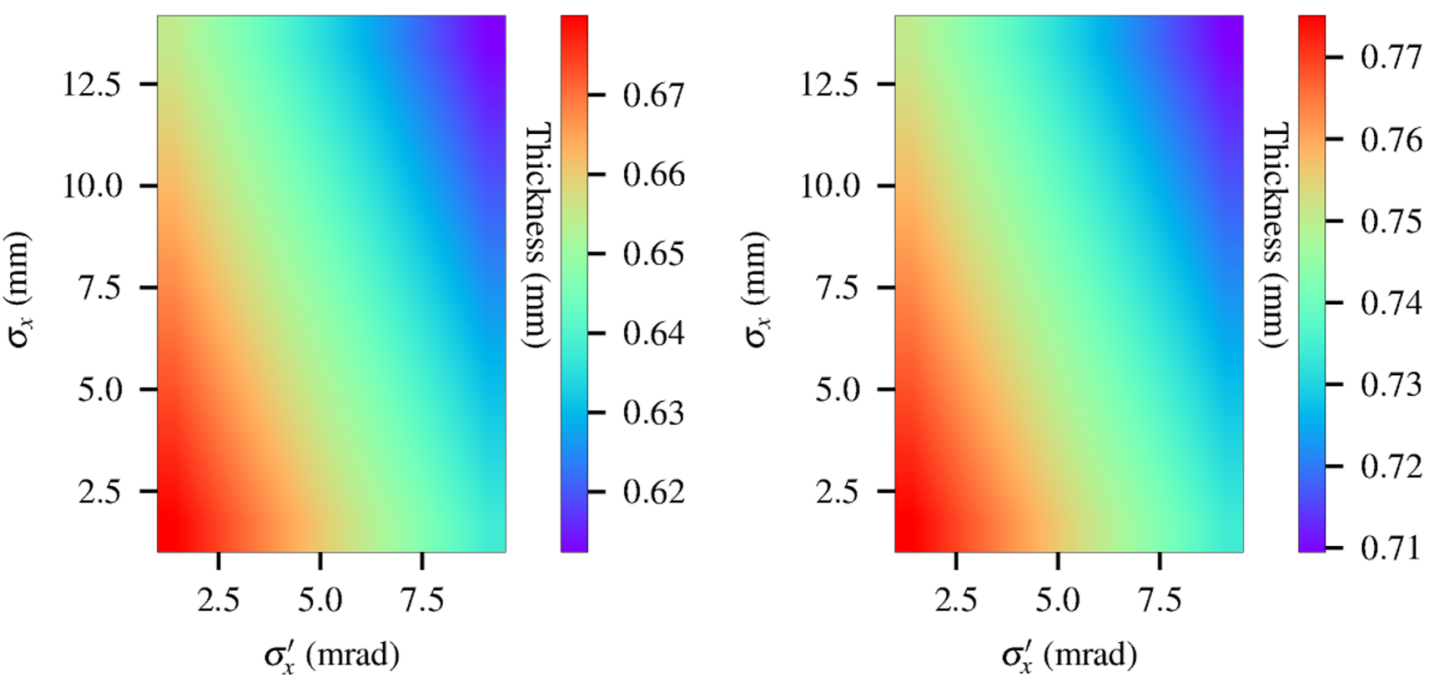

FIG. 4. Minimal required tantalum scatterer thickness to achieve a $2 \%$ transverse flatness at isocenter, as a function of the beam size and divergence, for two different values of the correlation parameter $A_{1}$. In the figure on the left, $A_{1}=10^{-4} \mathrm{~m}$ rad. In figure on the right, $A_{1}=-10^{-4} \mathrm{~m} \mathrm{rad}$.

level. Therefore, the flatness and dose rate criteria are closely related and must be fulfilled at the same time.

(iii) The lateral penumbra (both at skin and isocenter) is imposed by the scattering processes within the nozzle. It is also affected by the beam propagation distance from the exit of the aperture to isocenter.

With these complex machine parameters and dose profile properties interdependence, a preliminary step before designing the beamline is to study the impact of the most important beam and beamline parameters on the physical properties of the clinical beam. We conducted such a study through two sets of simulations. First, a semianalytical model was used to determine the minimum required scattering and range shifting foils that lead to a maximum $2 \%$ transverse flatness at isocenter. Second, Monte Carlo simulations were done using BDSIM to compute the depth dose profile DFO, as a function of the beam nominal energy $E$ and energy spread $(\Delta E / E)$.

As discussed in detail in [27], the electromagnetic interactions of charged particles in a uniform medium can be described by the Fermi-Eyges (FE) formalism. If $z$ is the propagation direction of a given charged particles beam and $(x, y)$ defines the transverse plane (i.e., the plane perpendicular to $z$ ), the FE formalism assumes a Gaussian distribution in each transverse phase space and gives the expression of the joint probability distribution $\mathrm{P}\left(x, \theta_{x}\right)\left(\mathrm{P}\left(y, \theta_{y}\right)\right)$ of finding a single particle at a transverse position $x(y)$, with a projected angle $\theta_{x}\left(\theta_{y}\right)$ :

$$
P(x, \theta)=\frac{1}{2 \pi \sqrt{A_{0} A_{2}-A_{1}^{2}}} e^{-\frac{A_{0} x^{2}-2 A_{1} x \theta+A_{2} \theta^{2}}{2\left(A_{0} A_{2}-A_{1}^{2}\right)}},
$$

where the quantities $A_{i}$ are the $i$ th order moments of the "scattering power" $T(z)$ of the propagation medium [27]. $T(z)$ quantifies the evolution of the variance of the projected angle $\theta$ as the beam propagates through the material. Equations (3a) and (3b) respectively give the definitions of $T(z)$ and the moments $A_{i}$ :

$$
\begin{aligned}
T(z) & =\frac{d\left\langle\theta^{2}\right\rangle}{d z} \\
A_{i}(z) & =\int_{0}^{z}(z-u)^{i} T(u) d u .
\end{aligned}
$$

The moments $A_{0}, A_{1}$, and $A_{2}$ define the beam optics in the transverse phase space, as they are respectively equal to the angular variance $\sigma_{\theta}^{2}$, transverse correlation $\sigma_{x \theta}$, and the spatial variance $\sigma_{x}^{2}$.

Using the FE formalism, we studied the impact of the beam distribution at the nozzle entrance on the required scattering and range shifting configurations in the nozzle to obtain the maximum of $2 \%$ transverse flatness. Several beam configurations (defined by their $A_{i}$ parameters) were propagated through the main components of the nozzle (tantalum scatterer, the Lexan range shifter, and the air gaps between the components). The model implemented allows for studying the effect of the beam-matter electromagnetic interactions on the beam's transverse distribution in both planes as a function of the incident nominal energy. We present in Fig. 4 the results obtained with a $70 \mathrm{MeV}$ incident beam, for two different values of the correlation moment $A_{1}$.

We can conclude from Fig. 4 that divergent $\left(A_{1}>0\right)$ and large transverse size and angular distribution beams lead to the smallest tantalum thicknesses. Therefore, during the beamline optimization, imposing such optics constraints on the beam distribution at the nozzle entrance will minimize the required scattering foil thickness and ultimately increase the beamline efficiency.

On the other hand, the impact of the nominal energy $E$ and energy spread $\Delta E / E$ on the depth dose deposition DFO must be studied to select the most appropriate energy and energy spread for the treatment. For this purpose, a BDSIM model was built, using a Gaussian beam which irradiates a $10 \times 10 \times 10 \mathrm{~cm}^{3}$ water phantom at different energies $E$ and 

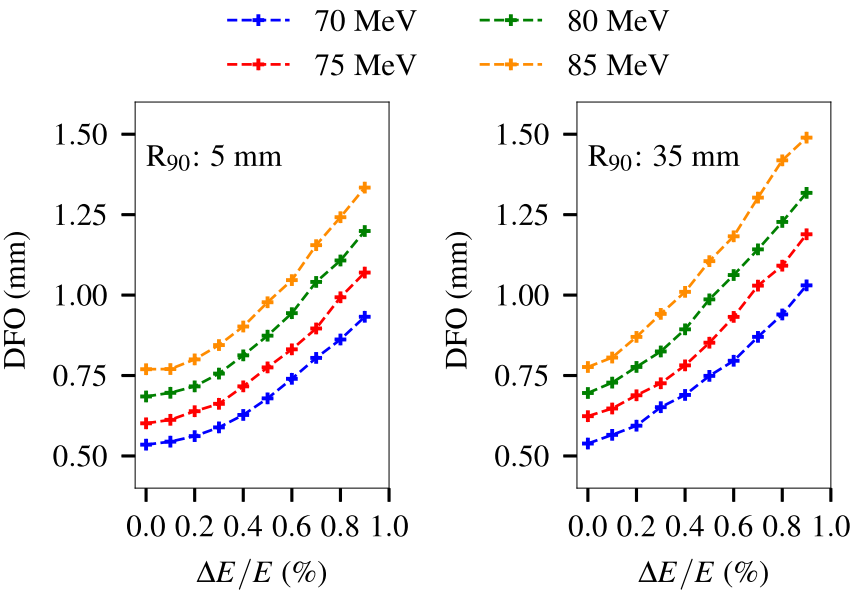

FIG. 5. Evolution of the DFO as a function of the beam nominal energy $E$ and energy spread $(\Delta E / E)$ for a range of $5 \mathrm{~mm}$ (on the left) and $35 \mathrm{~mm}$ (on the right).

energy spreads $\Delta E / E$. In this model, a Lexan range shifting slab is placed before the water phantom. This slab fixes the desired clinical range, regardless of the incoming beam energy. That way, simulations could be done for different clinical ranges, using the same pairs $(E, \Delta E / E)$. For each pair ( $E, \Delta E / E$ ), the DFO of the resulting depth dose deposition profile was computed, based on an eighth order polynomial fit on the distal region of the Bragg peak. The results obtained are shown in Fig. 5 for the minimum and the maximum required clinical ranges ( 5 and $35 \mathrm{~mm}$ ).

Figure 5 shows that the smallest values of both the nominal energy $E$ and the energy spread $\Delta E / E$ lead to the smallest DFO. On the other hand, we know that the transmission of the beamline (which is a consequence of the beam losses within the ESS) decreases with the beam nominal energy $E$. Moreover, a smaller beam energy spread $\Delta E / E$ allows a smaller DFO, but implies at the same time a lower beamline transmission. Therefore, the optimal pair $(E, \Delta E / E)$ for the treatment will always be a tradeoff between the limitation on the DFO and the desired beamline transmission, the latter being a factor that directly defines the achievable dose rate at isocenter.

\section{BEAMLINE OPTICS AND TRANSMISSION OPTIMIZATION}

The workflow proposed for the calibration of the IBA eyeline is made of three main steps, which are described in detail in Fig. 6. The first step is the optimization of the ESS. This optimization, which consists in setting the most appropriate optics with the quadrupoles Q1-7E and the opening of the momentum slits, allows one to accurately select the nominal energy of the beam at the exit of the degrader, while keeping the losses at the lowest possible level. The optimization of the beamline optics, which is the second step, consists in setting the currents of the remaining quadrupoles (Q8E to Q4F1) to maximize the global transmission (from the exit of the ESS to isocenter), with enough scattering and range shifting to guarantee the required transverse flatness and maximum range criteria. The last step of the workflow is the definition of the settings of the nozzle, to optimize the dosimetric properties of

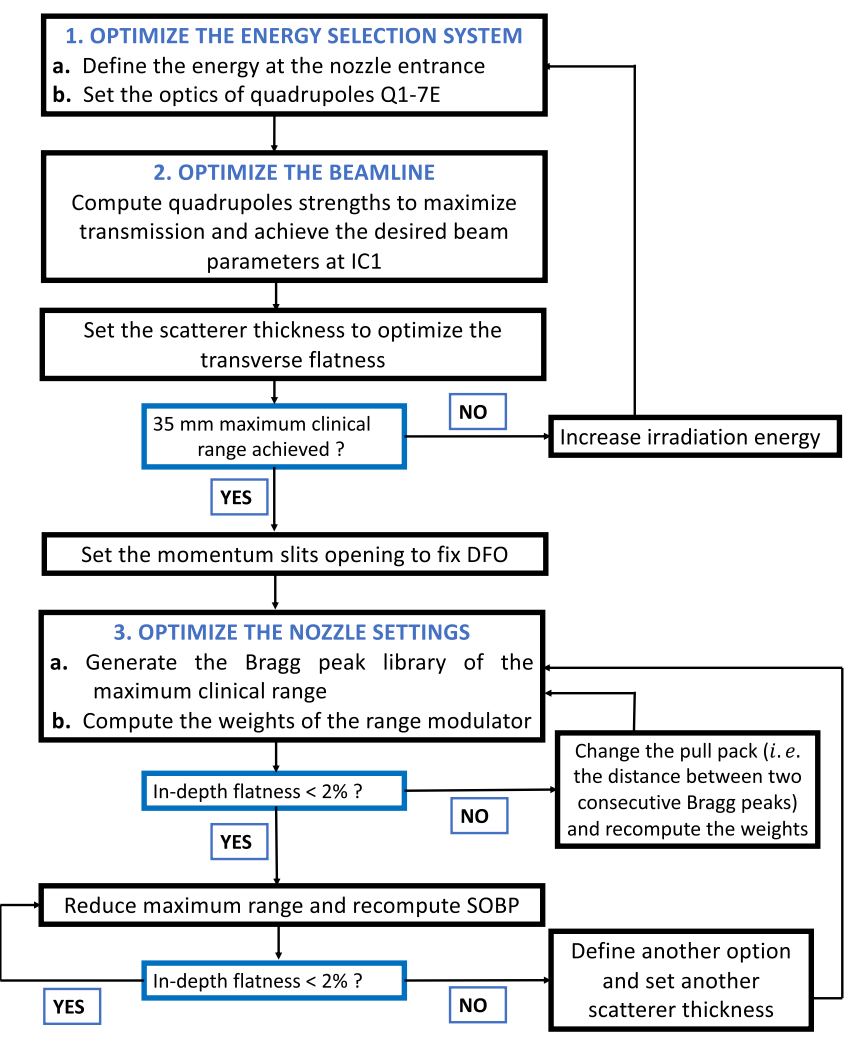

FIG. 6. Schematic detailed description of the calibration procedure of the IBA single scattering eye-treatment beamline. The procedure consists of optimizing the three main parts of the eyeline: the energy selection system (ESS), the beamline, and the nozzle.

the depth dose profiles, namely their flatness and DFO. The options of the eyeline are defined during this last step, with each option being defined by a given range interval, a fixed tantalum scatterer thickness, and a given RMW.

The first two steps of the workflow presented in Fig. 6 aim to define the beam nominal energy, to set the corresponding beamline optics (i.e., the magnets' currents), and to fix the thickness and the most appropriate position of the tantalum scatterer in the nozzle. As already presented in Table III, the resulting design must allow a maximum transverse flatness of $2 \%$, with a clinical range up to $35 \mathrm{~mm}$. We will present in this section the different steps to achieve these requirements. The third, final step of the calibration procedure, which consists in the detailed design of the nozzle settings, will be presented in Secs. V and VI.

The ESS (Q1-7E) is used to select the desired beam nominal energy $E$ and energy spread $\Delta E / E$, while keeping the losses at the lowest possible level, despite the large emittance increase induced by the degrader. It must manipulate the beam to be dispersion-dominated (minimal $\beta$ ) at the momentum slits to maximize the energy selection efficiency. It must also match into the rest of the transport beamline with zero horizontal dispersion $D_{x}$ and derivative $D_{x}^{\prime}$. For any solution, an independent constraint is that losses from aperture impacts should be minimized for maximum transmission efficiency. All these conditions give the following constraints for the design of the ESS: (1) maximize $D_{x}$ at the momentum slits location; (2) minimize $\beta_{x}$ at the momentum slits location; (3) 
TABLE IV. Final values obtained for the constraints' variables in the optics design of the ESS with a nominal energy of $80 \mathrm{MeV}$. mom stands for momentum slits, while max stands for maximum.

\begin{tabular}{lcccc}
\hline \hline $\begin{array}{l}D_{x}^{\text {mom }} \\
(\mathrm{m})\end{array}$ & $\begin{array}{c}\beta_{x}^{\text {mom }} \\
(\mathrm{m})\end{array}$ & $\begin{array}{c}\beta_{y}^{\text {mom }} \\
(\mathrm{m})\end{array}$ & $\begin{array}{c}\beta_{x}^{\max } \\
(\mathrm{m})\end{array}$ & $\begin{array}{c}\beta_{y}^{\max } \\
(\mathrm{m})\end{array}$ \\
\hline 1.99 & 0.49 & 0.79 & 20.04 & 18.06 \\
\hline
\end{tabular}

$D_{x}$ and $D_{x}^{\prime}$ equal to zero at the exit of the ESS; (4) $\beta_{x}$ and $\beta_{y}$ as small as possible all along the ESS.

The lowest energy achievable with the degrader wheel of the IBA Proteus ${ }^{\circledR}$ Plus system is $70 \mathrm{MeV}$. At this energy, the range of protons in water is $4.1 \mathrm{~cm}$, which is higher than the required maximum range for the eyeline $(35 \mathrm{~mm})$. On the other hand, we know that the beam-matter interaction processes with the scattering foil (which are required to obtain a transversally flat dose profile) inevitably induce nonnegligible range shifting. Moreover, the scattering in air along the nozzle is an additional source of range shifting for the clinical beam. Therefore, the most appropriate beam nominal energy must be selected carefully to guarantee a clinical range of at least $35 \mathrm{~mm}$, while keeping the transverse flatness lower than $2 \%$ at the same time.

Taking into account all these aspects, we selected a nominal energy of $80 \mathrm{MeV}$ for the beamline. We used our in-house particle tracking code to model the beamline, optimize the ESS, and find the most appropriate optical configuration of the quadrupoles Q1-7E to satisfy these constraints. ${ }^{2}$

The final values obtained for the constraints' variables with the optimization routine are summarized in Table IV. As can be seen, all the imposed constraints are satisfied. The horizontal dispersion $D_{x}$ reaches a maximum of $1.99 \mathrm{~m}$ at the momentum slits location. The horizontal and vertical $\beta$ functions exhibit a minimum at that same location, with maximal values of respectively $20.04 \mathrm{~m}$ and $18.06 \mathrm{~m}$ for $\beta_{x}$ and $\beta_{y}$, along the whole ESS (from Q1E to B4E). The resulting evolution of the $\beta$ functions and $D_{x}$ along the ESS is shown in Fig. 7.

For a given nominal energy, the maximum allowed momentum slits' opening is defined by the limitation on the DFO, given in Table III ( $<2 \mathrm{~mm}$ ). This maximum slits opening also put a limitation on the achievable beamline transmission (the higher the opening, the higher the transmission). Therefore, the beam losses need to be maintained at the lowest possible level along the rest of the beamline to guarantee a high dose rate. On the other hand, the semianalytical study presented in Sec. III showed that the beam parameters (size and divergence) at the entrance of the nozzle (IC1) have an impact on the minimal required scatterer thickness to achieve the $2 \%$ transverse flatness. Consequently, the ultimate dose rate (at isocenter) will depend not only on the losses that occur during the beam transport from the exit of the ESS to the nozzle entrance, but also on the losses that are due to the scattering processes within the nozzle. The transmission must therefore

\footnotetext{
${ }^{2}$ In our model, the opening of the divergence slits, which are located just before the first bending magnet (B1E), is set to $30 \mathrm{~mm}$ for all the simulations performed.
}
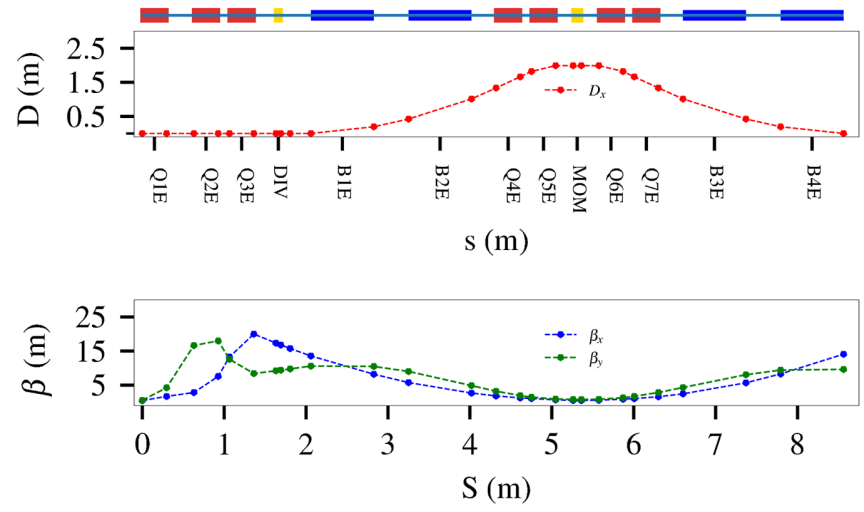

FIG. 7. Evolution of the horizontal dispersion $D_{x}$ (top) and the $\beta$ functions (bottom) along the ESS in the optimized configuration, for a nominal energy of $80 \mathrm{MeV}$.

be optimized over the complete beamline, including the nozzle, to satisfy all the requirements at the same time. This is why the second step of the optimization consists in maximizing the transmission from the exit of the ESS to isocenter, under the following constraints: (1) minimize the transverse asymmetry at the entrance of the nozzle; (2) minimize the beam sizes at the entrance of the nozzle; (3) minimize the transverse flatness at isocenter, which must be kept below $2 \%$.

The transverse asymmetry at the entrance of the nozzle $\alpha$ is defined by

$$
\alpha=\frac{\left|\sigma_{x}-\sigma_{y}\right|}{\sigma_{x}+\sigma_{y}} .
$$

We want a transversally symmetric beam at the nozzle entrance to guarantee the same field size in the two transverse axes (X and $\mathrm{Y}$ ) at isocenter after the scattering processes. The independent variables of this second optimization are the normalized gradients of the 10 remaining quadrupoles after the ESS (i.e., Q8E to Q4F1) and the thickness of the scatterer.

The values obtained for the constraints of the beamline optimization are summarized in Table V. The resulting evolution of the beam sizes along the beamline, obtained with $10^{6}$ primary protons at the degrader entrance, is shown in Fig. 8. The results obtained with our in-house tracking code are compared to simulations done with BDSIM. We can observe that BDSIM and our tracking code are in excellent agreement.

Table V clearly shows that the beam is symmetric at the entrance of the nozzle, with a transverse size of $9.8 \mathrm{~mm}$ in both $\mathrm{X}$ and $\mathrm{Y}$ axes. A $0.67 \mathrm{~mm}$ tantalum scatterer, combined with a $7.5 \mathrm{~mm}$ Lexan range shifter, allows one to set the

TABLE V. Values obtained for the constraints' variables at the end of the beamline optimization. The nominal energy of the beam at the exit of the degrader is $80 \mathrm{MeV}$. The transmission $\eta$ of the beamline, from the exit of the degrader to the entrance of the nozzle, is $7 \%$.

\begin{tabular}{lcccccc}
\hline \hline $\begin{array}{l}\sigma_{x} \\
(\mathrm{~mm})\end{array}$ & $\begin{array}{c}\sigma_{y} \\
(\mathrm{~mm})\end{array}$ & $\begin{array}{c}\alpha \\
/\end{array}$ & $\begin{array}{c}\text { Ta thick. } \\
(\mathrm{mm})\end{array}$ & $\begin{array}{c}\text { Le thick. } \\
(\mathrm{mm})\end{array}$ & $\begin{array}{c}\text { Flatness } \\
(\%)\end{array}$ & $\begin{array}{c}\eta \\
(\%)\end{array}$ \\
\hline 9.8 & 9.7 & $5.1 \times 10^{-3}$ & 0.67 & 7.5 & 1.91 & 7.0 \\
\hline \hline
\end{tabular}




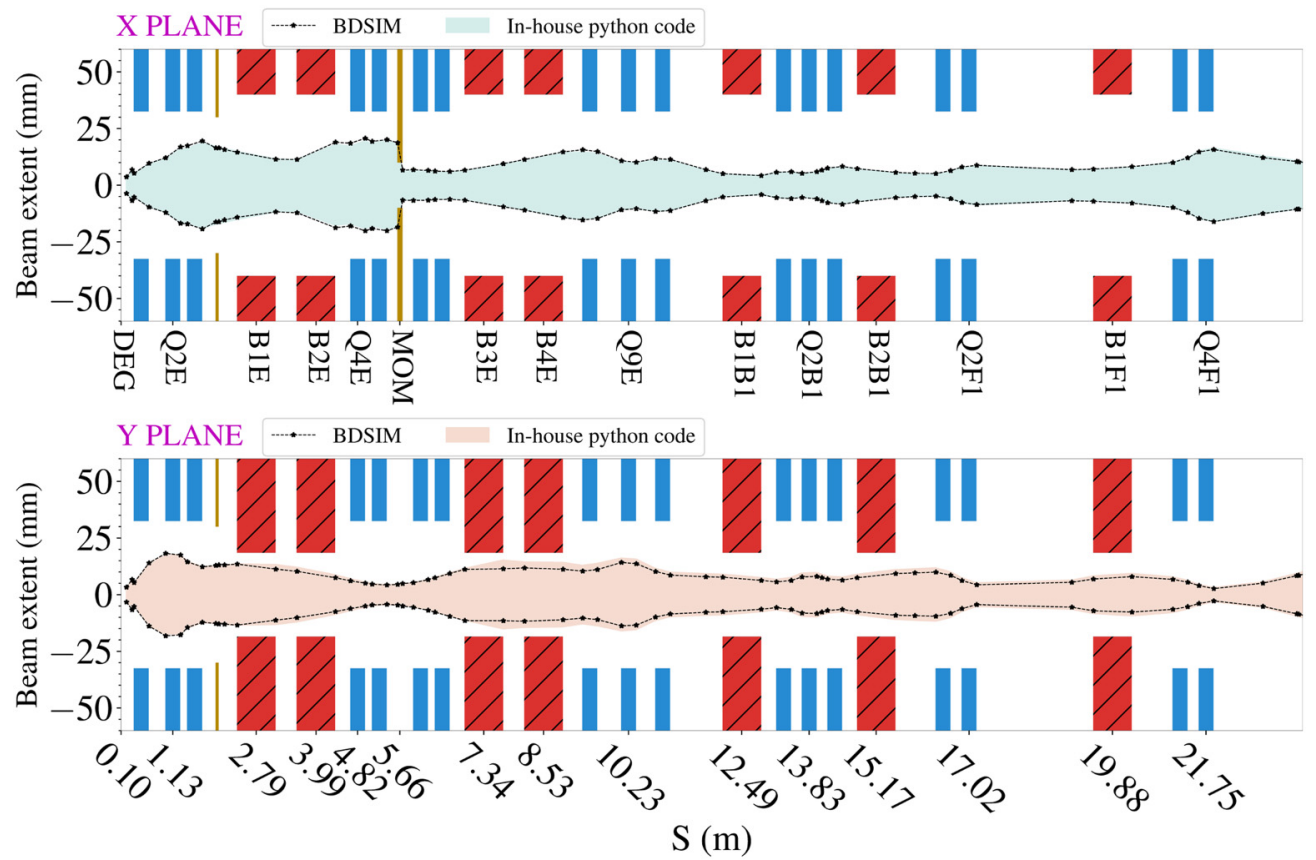

FIG. 8. Evolution of the beam transverse extents $(1 \sigma)$ in both $\mathrm{X}$ (above) and Y (bottom) axes, along the IBA passive scattering eyetreatment beamline, from the degrader to the entrance of the nozzle. The beam sizes obtained with our in-house tracking code are shown in cyan (for the $\mathrm{X}$ axis) and orange (for the $\mathrm{Y}$ axis), while BDSIM simulations results are visible in black. The nominal energy is set to $80 \mathrm{MeV}$. The horizontal (X) opening of the momentum limiting slits is set to $10 \mathrm{~mm}$. The two tracking codes exhibit an excellent agreement.

clinical range to $35 \mathrm{~mm}$, while keeping a transverse flatness of $1.91 \%$ for a nominal energy of $80 \mathrm{MeV}$. These dosimetric properties are visible in Fig. 9, where the transverse and depth dose profiles obtained with BDSIM are shown.

We can observe that the beam is perfectly symmetric on IC1. A flatness of $1.91 \%$ can be achieved with $0.67 \mathrm{~mm}$ of tantalum scatterer and the transmission of the beamline from the exit of the ESS to IC1 is $7.0 \%$.

Figure 10 presents the depth dose deposition profile obtained with the final design. We can observe that the DFO, which is defined by the momentum slits opening $(10 \mathrm{~mm})$, is equal to $1.7 \mathrm{~mm}(<2 \mathrm{~mm})$ and the maximum range is correctly fixed to $35 \mathrm{~mm}$.

\section{SYSTEM OPTIMIZATION TO ACHIEVE IMPROVED CLINICAL BEAM PROPERTIES}

The next step after the optimization of the beamline is the definition of the nozzle settings. The design of the nozzle consists in finding the most appropriate scattering and range shifting settings that can lead to the clinical performances summarized in Table III for all irradiation ranges (in the 5$35 \mathrm{~mm}$ interval).

The definition of the nozzle settings is mainly guided by the flatness of the depth dose profiles, regardless of the maximum clinical range. As typical eye tumor sizes range from 1.0 to $1.5 \mathrm{~cm}$, the depth dose deposition profiles need to
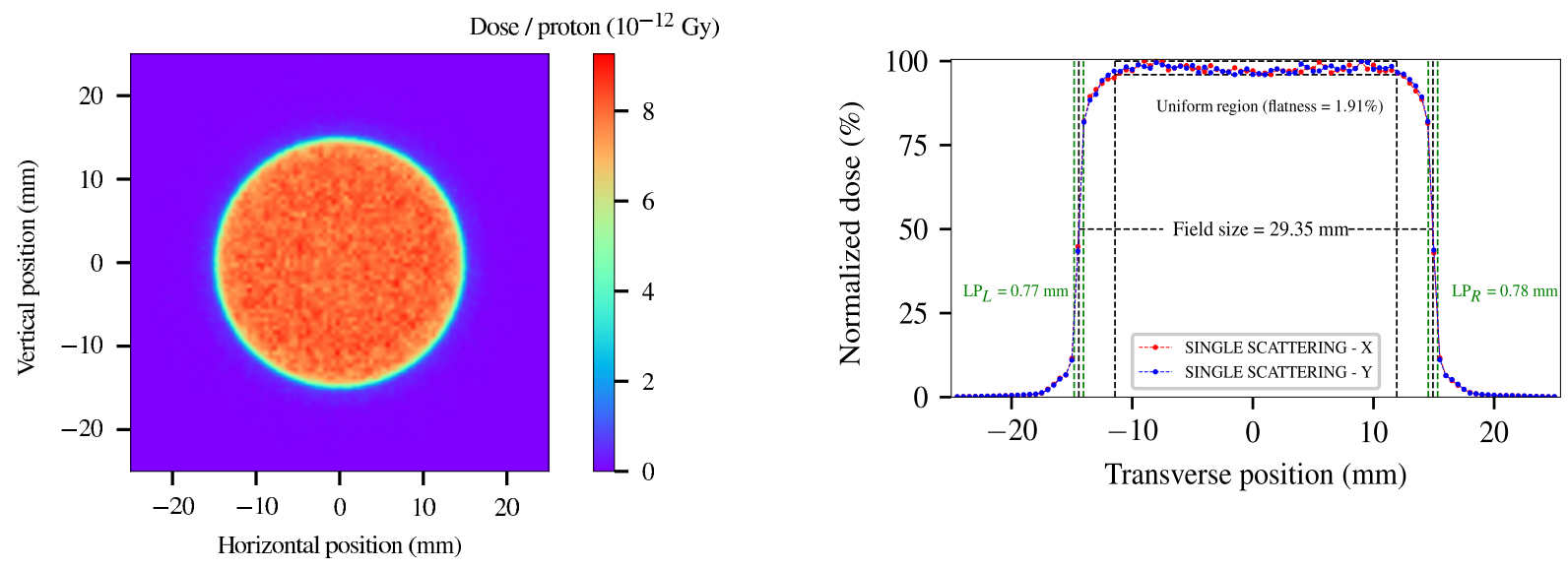

FIG. 9. 2D (on the left) and 1D (on the right) transverse dose profiles obtained with the optimized beamline, at a nominal energy of $80 \mathrm{MeV}$. The transverse flatness $(1.91 \%)$ and the lateral penumbra $(0.8 \mathrm{~mm})$ reach the clinical requirements. The $1 \mathrm{D}$ transverse profiles are shown for both the horizontal (X) and vertical (Y) axes. 


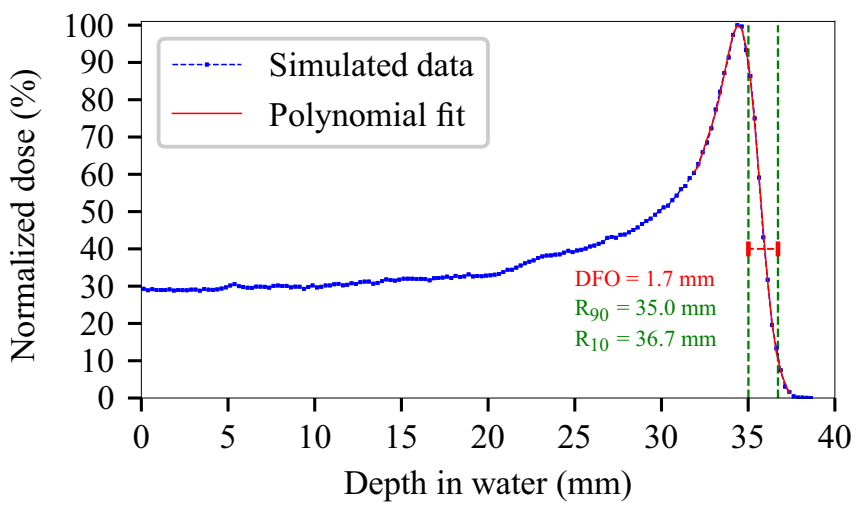

FIG. 10. Depth dose deposition profile obtained in single scattering mode for a nominal energy of $80 \mathrm{MeV}$. The maximum range is set to $35 \mathrm{~mm}$ using a $7.5 \mathrm{~mm}$ Lexan slab. The DFO, which is defined by the momentum slits opening $(10 \mathrm{~mm})$, is equal to $1.7 \mathrm{~mm}$.

be optimized to guarantee a flatness that is below the $2 \%$ clinical requirement, regardless of the maximum range and the thickness of the tumor. Such an in-depth coverage is achieved using the range modulation wheels (RMWs). RMWs are rotating wheels made of a stack of Lexan range shifting blocks with different thicknesses. During the interaction of the beam (which has a fixed nominal energy) with the different Lexan slabs, Bragg curves with different ranges are generated in the tumor, each peak being specific to a given Lexan thickness. A flat depth dose deposition profile can then be obtained by adjusting the relative weights of these different Bragg curves. In practice, this adjustment is done by setting the angular span of each step of the wheel.

We must notice that all the range shifting processes (that occur during the interaction with the range shifter and the RMW) also imply scattering and, at the end, beam losses. Consequently, range shifting and scattering processes need to be taken into account at the same time during the nozzle settings optimization. To be able to adjust the scatterer and the range shifter thicknesses at the same time, the minimal required scatterer thickness was computed as a function of the required clinical range, the latter being adjusted with the appropriate range shifter thickness. We present the results obtained in Fig. 11 for a nominal energy of $80 \mathrm{MeV}$ at the entrance of the nozzle.

Figure 11 shows that the required tantalum scatterer thickness decreases with the desired clinical range. This result is expected as a lower clinical range requires a thicker Lexan range shifter, which in turn implies more scattering contribution from this range shifter and, at the end, a reduced thickness of the scattering foil is required. The ideal way to minimize beam losses for all ranges (in the 5-35 mm interval) would be a systematic modification of the scatterer thickness as a function of the desired clinical range so that the dose rate (DR) remains almost constant over the whole range span. This solution is unfortunately not possible in practice, as it would imply a dynamic adaptation of the scattering foil thickness, not only to the range shifter thickness, but also to each of the individual Bragg peaks generated by the RMW. Therefore, at this stage, the scatterer thickness must be kept constant for a

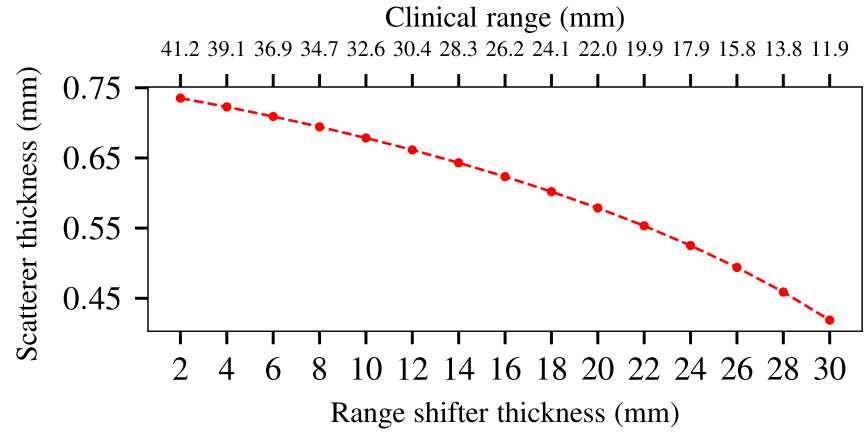

FIG. 11. Evolution of the minimal required tantalum scatterer thickness (to achieve $2 \%$ flatness) as a function of the desired clinical range at isocenter, for the same $(80 \mathrm{MeV})$ nominal energy beam at the entrance of the nozzle. The Lexan range shifting foil thickness required for each clinical range is also indicated.

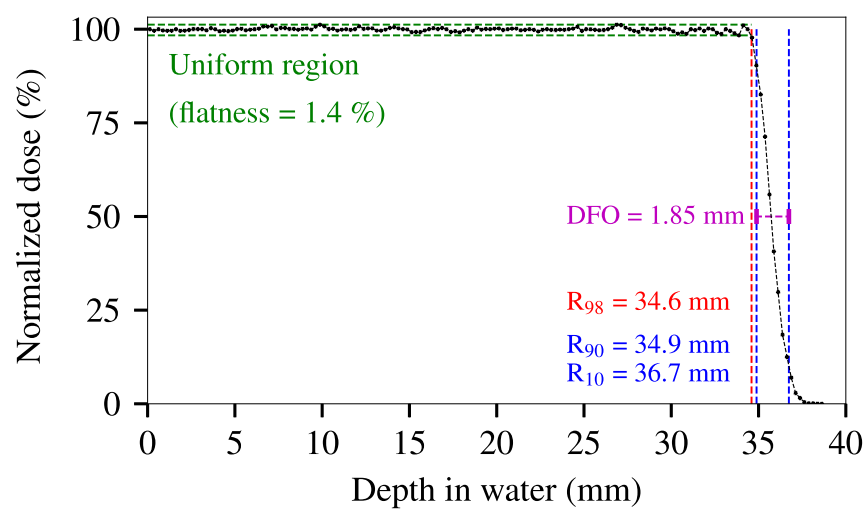

FIG. 12. Spread-out Bragg peak (SOBP) computed for a maximum clinical range of $35 \mathrm{~mm}$. The in-depth flatness is $1.4 \%$, which is below the $2 \mathrm{~mm}$ criterion. The DFO (equal to $1.85 \mathrm{~mm}$ ) is also below the $2 \mathrm{~mm}$ requirement.

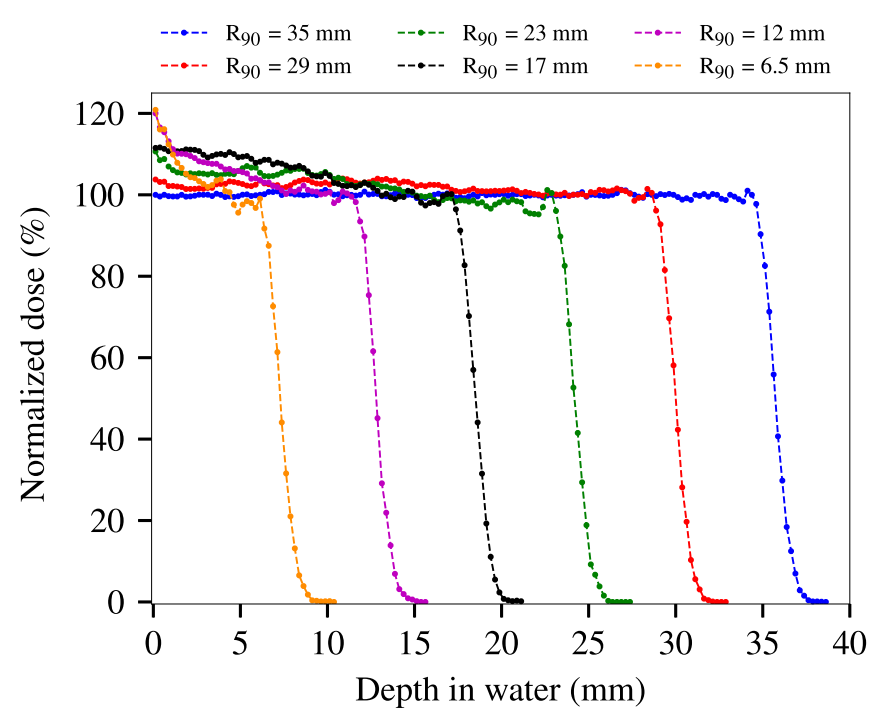

FIG. 13. Illustration of the tilt of the SOBP profile when the same wheel is used for different ranges to generate the depth dose profile. The minimal range of a given option is the value at which the flatness of the SOBP is higher than the $2 \%$ requirement. 


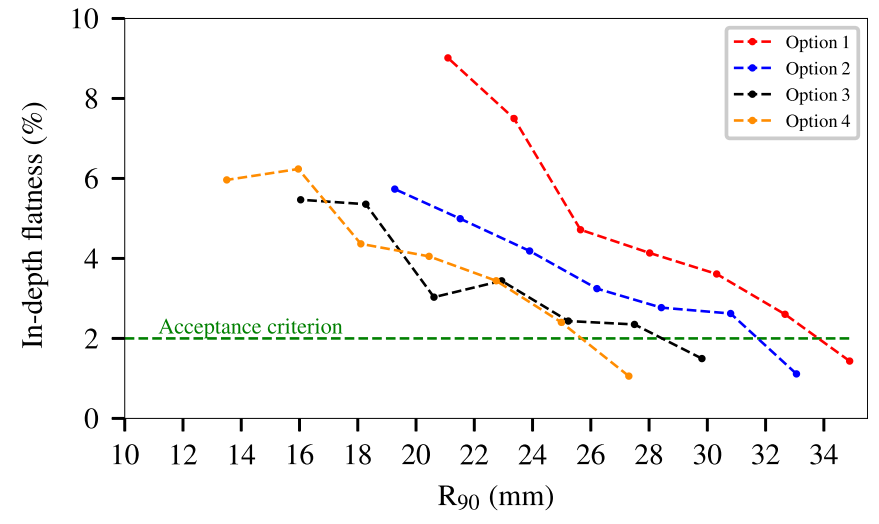

FIG. 14. Evolution of the in-depth flatness as a function of the clinical range for the first four options of the IBA passive scattering eye-treatment beamline in single scattering mode. The $2 \mathrm{~mm}$ acceptance criterion is highlighted with a dashed green line. The maximum clinical range of each option is defined from the intersection point of the in-depth flatness curve of the previous option, with this acceptance line.

given RMW and must be chosen related to the highest range, to always guarantee a flat transverse dose profile.

BDSIM simulations were performed to score depth dose deposition profiles starting from the maximum range $(35 \mathrm{~mm})$, and progressively increasing the thickness of the Lexan range shifter per $1 \mathrm{~mm}$ step, to generate the first "Bragg peak library." The resulting depth dose deposition profile [the spread-out Bragg peak (SOBP)] is reconstructed by weighting the individual Bragg peaks and is presented in Fig. 12.
As can be seen from Fig. 12, the uniform region, here defined as the region from skin to the depth at which the SOBP reaches $98 \%$ of its maximal value in the distal edge, has a flatness of $1.4 \%$. The DFO of the SOBP is $1.85 \mathrm{~mm}$ and the maximum range is equal to $35 \mathrm{~mm}$.

From this SOBP and the related weights, the evolution of the in-depth flatness as a function of the maximum range can now be computed, using the same weights as computed for a range of $35 \mathrm{~mm}$, to highlight the fact that the required relative weights are specific to the SOBP clinical range. Indeed, using the same weights for lower ranges inevitably leads to a "tilt" of the SOBP profile, and finally results into an in-depth flatness which is higher than the requirement of $2 \%$. Figure 13 illustrates the variation of the in-depth flatness with the clinical range when the same wheel is used to compute the $\mathrm{SOBP}$, regardless of the maximum range.

As this flatness must always be kept below the $2 \%$ requirement, the range at which the criterion is not reached anymore is the minimum range at which we can use the designed wheel. For lower ranges, we need to generate another SOBP, and recompute the weights to allow a flatness lower than $2 \%$ again. Therefore, the strategy consists in splitting the range span into several options. An option is defined by a given clinical range span and is associated to a fixed tantalum scatterer thickness and a specific RMW. From one option to another, we can reduce the scatterer thickness according to Fig. 11 for lower ranges, so that the dose rate can be kept almost constant over all the ranges interval. For each option, the fixed scatterer thickness corresponds to the minimum required value for the highest range, to always maintain the transverse flatness below
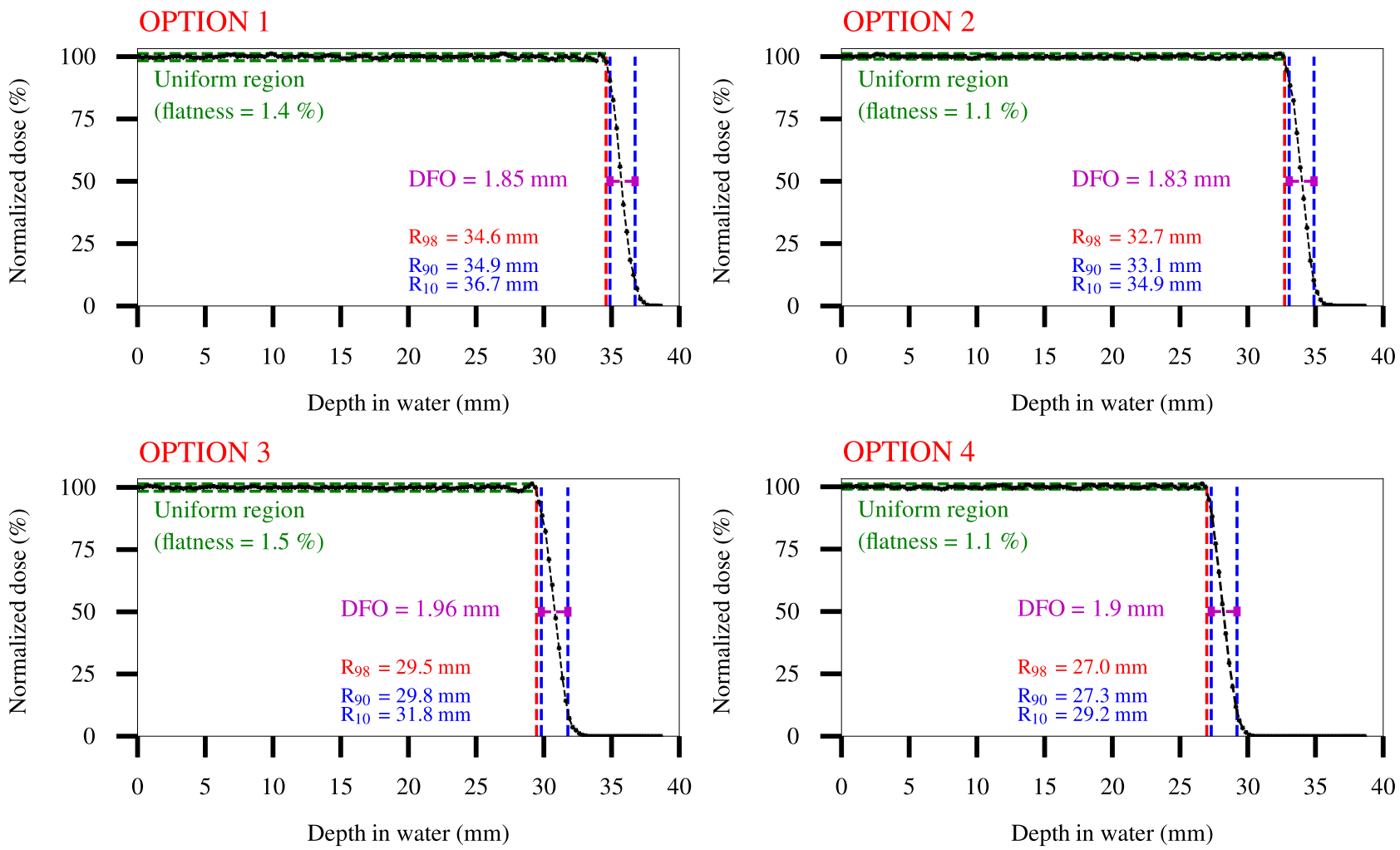

FIG. 15. Full modulation depth dose deposition profiles obtained for the first four options of the beamline, in single scattering mode. 
TABLE VI. Minimum and maximum ranges, the tantalum scatterer thickness, the minimum Lexan thickness, the DFO, the in-depth flatness, and the dose rates obtained for the 12 options defined in single scattering mode.

\begin{tabular}{|c|c|c|c|c|c|c|c|c|}
\hline Option & $\begin{array}{l}\mathrm{R}_{\max } \\
(\mathrm{mm})\end{array}$ & $\begin{array}{l}\mathrm{R}_{\min } \\
(\mathrm{mm})\end{array}$ & $\begin{array}{l}\text { Ta thick. } \\
\text { (mm) }\end{array}$ & $\begin{array}{l}\text { Min. Le thick. } \\
\qquad(\mathrm{mm})\end{array}$ & $\begin{array}{l}\text { DFO } \\
(\mathrm{mm})\end{array}$ & $\begin{array}{c}\text { Flatness } \\
(\%)\end{array}$ & $\begin{array}{c}\mathrm{DR}_{\max } \\
(\mathrm{Gy} / \mathrm{min})\end{array}$ & $\begin{array}{c}\mathrm{DR}_{\min } \\
(\mathrm{Gy} / \mathrm{min})\end{array}$ \\
\hline 1 & 35.0 & 33.1 & 0.67 & 7.5 & 1.85 & 1.40 & 16.0 & 15.1 \\
\hline 2 & 33.1 & 29.8 & 0.60 & 9.5 & 1.83 & 1.10 & 16.5 & 15.2 \\
\hline 3 & 29.8 & 27.3 & 0.55 & 12.5 & 1.96 & 1.50 & 16.3 & 15.3 \\
\hline 4 & 27.3 & 25.3 & 0.55 & 15.2 & 1.90 & 1.10 & 17.0 & 15.7 \\
\hline 5 & 25.3 & 20.5 & 0.45 & 17.2 & 1.83 & 1.53 & 17.0 & 15.9 \\
\hline 6 & 20.5 & 18.5 & 0.40 & 19.5 & 1.68 & 1.75 & 16.0 & 15.0 \\
\hline 7 & 18.5 & 16.3 & 0.35 & 21.0 & 1.64 & 1.81 & 16.5 & 15.0 \\
\hline 8 & 16.3 & 14.0 & 0.30 & 23.3 & 1.71 & 1.52 & 16.7 & 15.2 \\
\hline 9 & 14.0 & 11.7 & 0.25 & 25.0 & 1.60 & 1.27 & 16.2 & 15.2 \\
\hline 10 & 11.7 & 9.5 & 0.20 & 26.8 & 1.59 & 1.31 & 15.5 & 15.1 \\
\hline 11 & 9.5 & 7.0 & 0.15 & 28.0 & 1.61 & 1.17 & 16.0 & 15.3 \\
\hline 12 & 7.0 & 5.0 & 0.10 & 29.5 & 1.77 & 1.45 & 15.7 & 15.0 \\
\hline
\end{tabular}

the $2 \%$ limitation. However, this configuration implies that the lower ranges within the same option will be overscattered due to the required additional range shifter and will finally have a slightly smaller dose rate.

To illustrate the principle of options definition, we present in Fig. 14 the evolution of the in-depth flatness as a function of the range, for the first four options.

Based on this principle, 12 options were defined for the single scattering design. We present in Fig. 15 the SOBP dose profile obtained for these first four options.

For each option, the maximum achievable dose rate can be estimated from the cyclotron beam current $\mathrm{I}_{\text {cyclo }}$ and the transmission of the beamline $\eta_{\text {beamline }}$ using Eq. (5). The beamline transmission $\eta_{\text {beamline }}$ is given by the ratio between the number of protons on IC1 $\mathrm{N}_{I C_{1}}$ divided by the number of protons at the degrader entrance $\mathrm{N}_{d e g}$. The cyclotron current $\mathrm{I}_{\text {cyclo }}$, which was initially $300 \mathrm{nA}$, has been upgraded to $500 \mathrm{nA}$ to allow a higher dose rate. This latter value has been used to compute

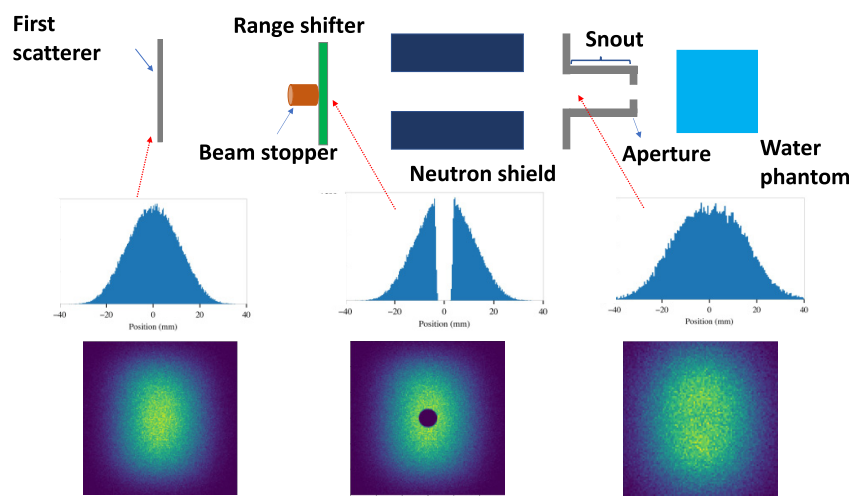

FIG. 16. Transverse beam forming principle of the beam stopper device combined with a thin tantalum scatterer. The particles first get a small kick when interacting with the scatterer; then a hole is created at the center of the beam by the beam stopper. This "hole" is progressively filled during the propagation along the nozzle and a flat transverse profile is obtained at isocenter. the dose rates of all defined options, as follows:

$$
\mathrm{DR}_{\max }=\frac{\mathrm{I}_{\text {cyclo }}}{e} \times \eta_{\text {beamline }} \times \mathrm{D}_{\text {max }} .
$$

In Eq. (5), $\mathrm{D}_{\max }$ is the maximum deliverable dose per proton and $e$ is the elementary charge of a proton. It is the maximum value of the pristine Bragg peak at the maximum clinical range of the considered option. We summarize in Table VI the final values obtained for the minimum and maximum ranges, the DFO, the in-depth flatness, and the minimum and maximum dose rates for all defined options.

From Table VI, we can observe that all options have a DFO below $2 \mathrm{~mm}$ and an in-depth flatness lower than $2 \%$. The overall maximum dose rate is $17.5 \mathrm{~Gy} / \mathrm{min}$, while the minimum dose rate is $15.1 \mathrm{~Gy} / \mathrm{min}$. In practice, each treatment option is characterized by a specific range modulation wheel. The wheels are manufactured according to the relative weights of the pristine peaks simulated to construct the SOBPs. At this stage, the eyeline is optimized in single scattering mode and all the clinical requirements are fulfilled. Nevertheless, the low transmission of the nozzle is responsible for a limited maximum achievable dose rate.

\section{NOZZLE DESIGN USING A BEAM STOPPER DEVICE FOR ENHANCED DOSE RATE}

The main disadvantage of the single scattering solution presented in Sec. V is the transmission of the nozzle, which turns out to be too low given the required scatterer thickness to achieve the $2 \%$ transverse flatness. The maximum achievable dose rate was consequently limited to $17 \mathrm{~Gy} / \mathrm{min}$.

This maximum dose rate can be increased with an alternative beam spreading mechanism, which combines a very thin scattering foil with a cylindrical high $Z$ material beam stopper as shown in Fig. 16. As can be seen, the beam stopper absorbs particles due to its higher $Z$ and density than water and biological materials. The transverse hole created in the beam distribution is gradually filled in as the beam propagates, resulting in a flat transverse beam profile at isocenter. 

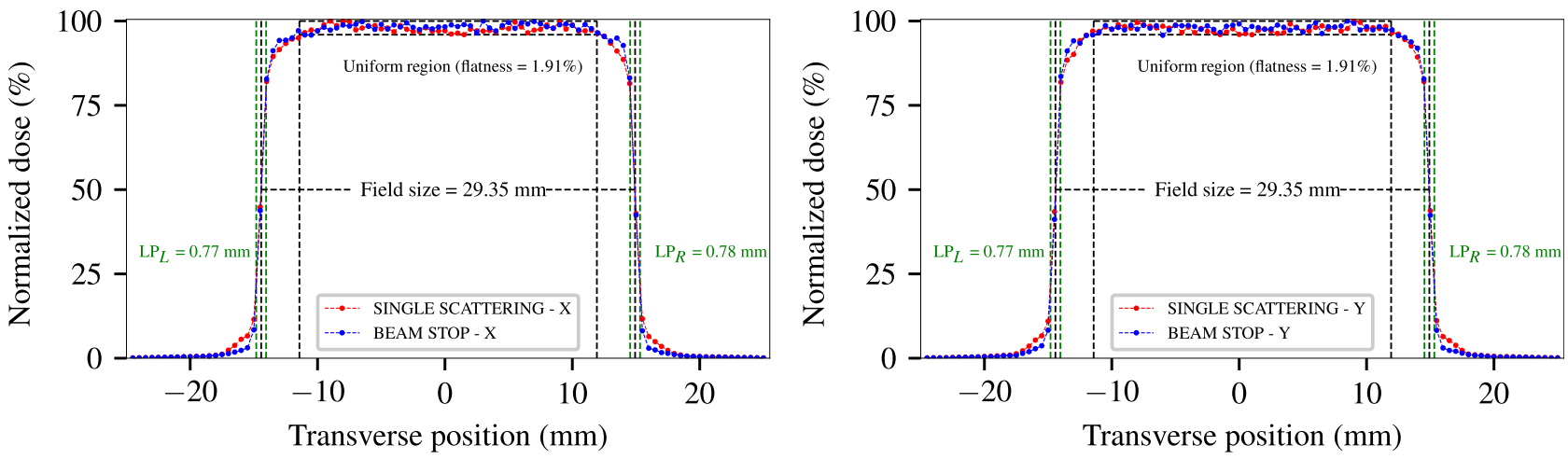

FIG. 17. Lateral profiles along the horizontal (left) and vertical (right) axes for the beam stopper design in blue, compared to the single scattering design in red. The transverse flatness and lateral penumbra are indicated.

Such a combination ${ }^{3}$ requires less scattering material (typically less than $200 \mu \mathrm{m}$ ) to achieve a flat transverse dose profile. Consequently, the transmission is higher and a higher dose rate is achievable. The design consists in determining the most appropriate thickness, together with the required beam stopper radius and position, so that the flatness requirement can be satisfied with a maximum nozzle transmission. Starting with a $10-\mathrm{mm}$-thick lead beam stopper, with a radius of $3 \mathrm{~mm}$, placed at a distance of $30 \mathrm{~cm}$ from the scatterer, we adjusted all of these parameters so that the clinical requirements could be reached at isocenter, while maintaining the losses at the lowest possible level. We summarize in Table VII the final values obtained for all the design parameters, for a $80 \mathrm{MeV}$ beam at the nozzle entrance.

As shown in Table VII, the scatterer thickness is only $0.15 \mathrm{~mm}$, which is four times smaller than the required value for the single scattering mode at the same clinical range $(0.67 \mathrm{~mm})$. Combining this thin scatterer with a lead, $10 \mathrm{~mm}$ thick, cylindrical beam stopper, positioned $35.5 \mathrm{~cm}$ downstream of the scatterer, leads to a uniform transverse profile at isocenter. We also observe from Table VII that a $12 \mathrm{~mm}$ Lexan range shifter is needed to set the maximum clinical range to $35 \mathrm{~mm}$. It would have been possible to avoid this additional range shifting by decreasing the nominal energy at the entrance of the nozzle, but this alternative solution would

\footnotetext{
${ }^{3}$ To the best of our knowledge, the only usage of a beam stopper device in a proton therapy center dedicated to ocular cancer treatment is at the Charité Hospital, which treats eye cancer patients with a dedicated, lower energy proton therapy beamline, in collaboration with the Helmholtz-Zentrum in Berlin, Germany.
}

TABLE VII. Design parameters of the nozzle when used with a beam stopper device. A 12-mm-thick Lexan range shifter, placed just downstream of the beam stopper, is used to fix the maximum clinical range to $35 \mathrm{~mm}$.

\begin{tabular}{lccccc}
\hline \hline $\begin{array}{l}\text { Ta thick. } \\
(\mathrm{mm})\end{array}$ & $\begin{array}{c}\text { Le thick. } \\
(\mathrm{mm})\end{array}$ & $\begin{array}{c}\text { Material } \\
/\end{array}$ & $\begin{array}{c}\text { Radius } \\
(\mathrm{mm})\end{array}$ & $\begin{array}{c}\text { Thick. } \\
(\mathrm{mm})\end{array}$ & $\begin{array}{c}\mathrm{D}_{\mathrm{FS}-\mathrm{BS}} \\
(\mathrm{cm})\end{array}$ \\
\hline 0.15 & 12 & Lead & 3.6 & 10 & 35.5 \\
\hline \hline
\end{tabular}

mean another optimization of the beamline, at the selected nominal energy.

Figure 17 shows the lateral dose profiles obtained with the final design, in the horizontal and vertical axes, including the profiles obtained in single scattering mode. We can observe that the shapes of the lateral profiles are very similar. The lateral penumbra and the transverse flatness are the same along both axes.

The depth dose deposition profiles of the two designs were also simulated and are compared in Fig. 18. From the plot on the left, we observe that the dose per proton at the entrance of the nozzle obtained with the beam stopper is three times higher than the value obtained in single scattering mode. From the plot on the right we observe that the beam stopper design exhibits a lower dose at skin, from which the patient can benefit in terms of sparing OARs. The DFO, the lateral penumbra (LP), the maximum dose per proton at the entrance of the nozzle, and the dose at skin of the two designs are summarized and compared in Table VIII.

As for the single scattering design, BDSIM simulations were performed to generate the Bragg peak libraries using the beam stopper. For each option defined, the maximum dose rate, the in-depth flatness, and the DFO were computed. The results obtained are summarized in Table IX.

We can conclude that the use of a beam stopper device, in combination with a thin first scatterer, shows better clinical performances than the single scattering design, especially in terms of dose rate. The maximum achievable dose rate is $42.4 \mathrm{~Gy} / \mathrm{min}$ (i.e., $0.7 \mathrm{~Gy} / \mathrm{s}$ ) for a maximum cyclotron current of $500 \mathrm{nA}$. This value decreases with the clinical range, due to the additional range shifter required for the lower ranges. Indeed, the lower the range, the lower the maximum value of the Bragg peak as shown in Fig. 19.

TABLE VIII. Comparison of the clinical performances of the single scattering and the beam stopper designs.

\begin{tabular}{lccccc}
\hline \hline Design & $\begin{array}{c}\text { DFO } \\
(\mathrm{mm})\end{array}$ & $\begin{array}{c}\text { LP } \\
(\mathrm{mm})\end{array}$ & $\begin{array}{c}\text { Flatness } \\
(\%)\end{array}$ & $\begin{array}{c}\mathrm{D}_{\max } \\
(\mathrm{Gy} / \text { prim. })\end{array}$ & $\begin{array}{c}\mathrm{D}_{\text {skin }} \\
\left(\% \text { of } \mathrm{D}_{\text {max }}\right)\end{array}$ \\
\hline SS & 1.65 & 0.78 & 1.90 & $0.56 \times 10^{-11}$ & 30.0 \\
BS & 1.66 & 0.77 & 1.91 & $1.57 \times 10^{-11}$ & 25.0 \\
\hline \hline
\end{tabular}



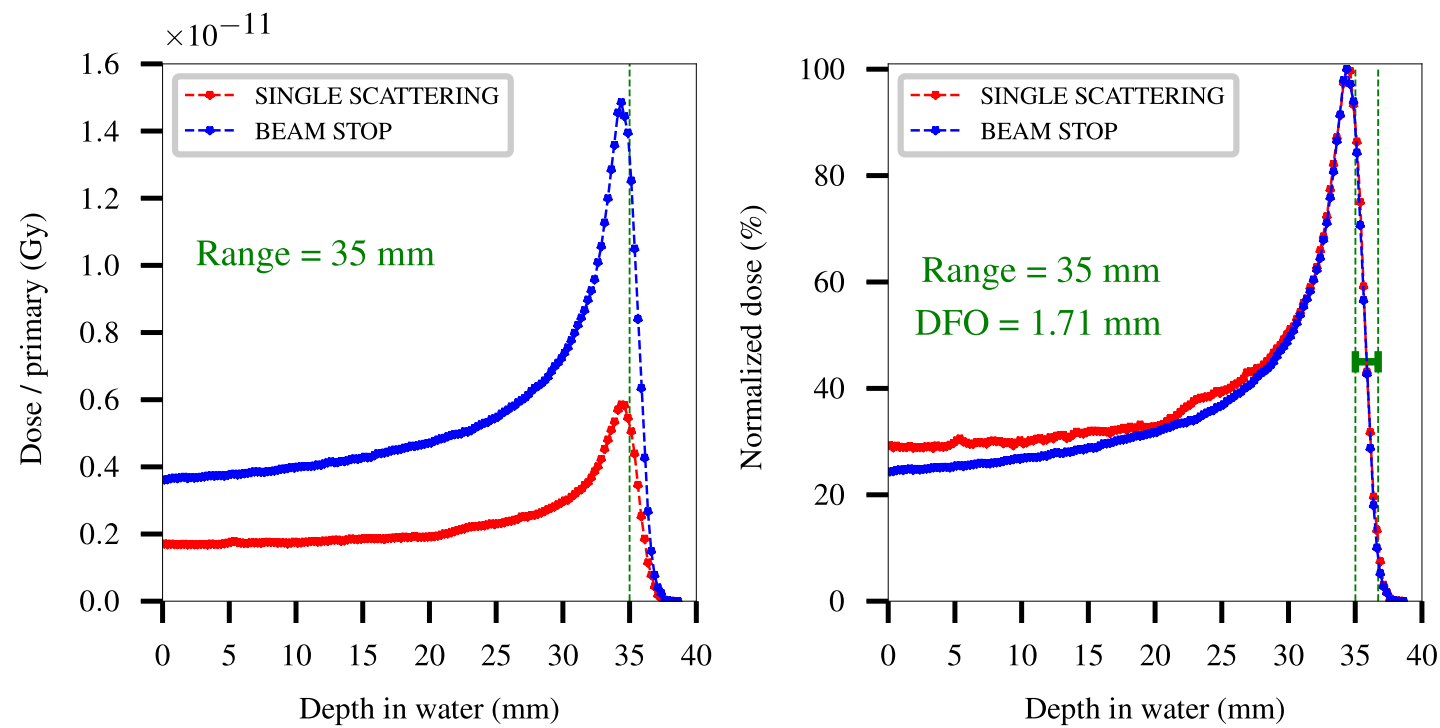

FIG. 18. Depth dose profiles obtained for the beam stopper design in blue, compared to the single scattering design in red. The plot on the left shows that the dose per proton at the entrance of the nozzle obtained with the beam stopper is three times higher than the value obtained in single scattering mode.

To schematically illustrate the evolution of the dose rate as a function of the irradiation range, we represent in Fig. 20 the minimum and maximum dose rates of each of the 12 options defined, both in single scattering mode and with the beam stopper. Each option is represented by a rectangular box, limited by the minimum and maximum ranges (on the left and on the right, respectively) and the minimum and maximum dose rates (on the bottom and on the top, respectively). We observe that the higher the range, the higher the benefit of the beam stopper in terms of dose rate.

\section{CONCLUSION}

The complete optimization of low energy, passive scattering proton therapy systems to achieve well-defined dose deposition profiles, which can cover small and shallow treatment volumes like eye tumors, while efficiently sparing the surrounded OARs, presents many challenges. This paper presents a numerical approach to tackle the step-by-step optimization of such passive scattering systems in the specific context of ocular tumors treatment.

The 3D model of the IBA Proteus ${ }^{\circledR}$ Plus passive scattering eye-treatment beamline, programatically built with BDSIM, was presented in detail in Sec. II. The model includes the beam transport system (from the exit of the accelerator to the entrance of the treatment room), the beam forming nozzle, and the main shielding walls of the treatment room. The features of its main components were discussed to highlight the close links between several machine parameters, the physical properties of the delivered beam, and the resulting clinical performances. In Sec. III, those links were quantified, first based on a study of the influence of the beam optics on the required scatterer thickness to obtain a maximum transverse flatness of $2 \%$ at isocenter. Following this study, a set of Monte Carlo simulations was done to assess the variation of the depth dose profiles DFO as a function of the beam energy

TABLE IX. Minimum and maximum ranges, the minimum Lexan range shifter thickness, the DFO, the in-depth flatness, and the dose rates obtained for the 12 options defined for the beam stopper design.

\begin{tabular}{lccccccr}
\hline \hline Option & $\begin{array}{c}\mathrm{R}_{\max } \\
(\mathrm{mm})\end{array}$ & $\begin{array}{c}\mathrm{R}_{\min } \\
(\mathrm{mm})\end{array}$ & $\begin{array}{c}\text { Min. Le thick. } \\
(\mathrm{mm})\end{array}$ & $\begin{array}{c}\mathrm{DFO} \\
(\mathrm{mm})\end{array}$ & $\begin{array}{c}\text { Flatness } \\
(\%)\end{array}$ & $\begin{array}{c}\mathrm{DR}_{\max } \\
(\mathrm{Gy} / \mathrm{min})\end{array}$ & $\begin{array}{r}\mathrm{DR}_{\min } \\
(\mathrm{Gy} / \mathrm{min})\end{array}$ \\
\hline 1 & 35.0 & 31.5 & 12.0 & 1.80 & 1.60 & 42.4 & 38.6 \\
2 & 31.5 & 28.8 & 14.9 & 1.80 & 1.10 & 38.6 & 34.7 \\
3 & 28.8 & 27.0 & 17.2 & 1.70 & 1.50 & 34.7 & 34.2 \\
4 & 27.0 & 25.5 & 18.7 & 1.82 & 1.50 & 34.2 & 31.7 \\
5 & 25.5 & 23.5 & 20.0 & 1.86 & 1.85 & 31.7 & 29.4 \\
6 & 23.5 & 20.7 & 21.6 & 1.82 & 0.96 & 29.4 & 27.1 \\
7 & 20.7 & 18.5 & 24.1 & 1.75 & 1.38 & 27.1 & 24.0 \\
8 & 18.5 & 17.0 & 25.8 & 1.83 & 1.56 & 25.2 & 20.0 \\
9 & 17.0 & 12.0 & 27.0 & 1.68 & 1.70 & 24.0 & 18.5 \\
10 & 12.0 & 9.5 & 31.0 & 1.57 & 1.63 & 20.0 & 17.5 \\
11 & 9.5 & 8.0 & 33.2 & 1.75 & 1.56 & 18.5 & 17.5 \\
12 & 8.0 & 5.0 & 34.5 & 1.51 & 1.43 & & \\
\hline \hline
\end{tabular}




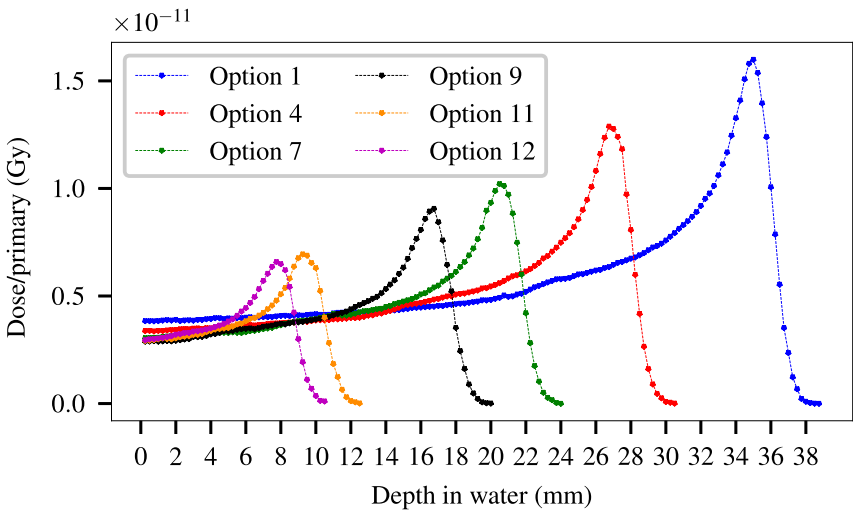

FIG. 19. Dose deposition profiles for the maximum ranges of different options with the beam stopper design. The maximum of the Bragg curves decreases with the range.

and energy spread. The simulations show that a high nominal energy, together with a low energy spread and divergent beam, is desirable to obtain the best clinical performances (i.e., small DFO and limited losses within the nozzle). The optimization of the ESS and the beamline transmission led to the selection of a nominal energy of $80 \mathrm{MeV}$ at the nozzle entrance. At this energy, the $35 \mathrm{~mm}$ maximum range and the $2 \%$ transverse flatness clinical requirements were satisfied with a combination of a $0.67 \mathrm{~mm}$ tantalum scatterer and a $7.5 \mathrm{~mm}$ Lexan range shifter.

The detailed design of the settings of the nozzle in single scattering mode was presented in Sec. V and resulted into the definition of 12 treatment options. BDSIM simulations were performed to compute the clinical properties of the full modulation SOBP of each option, namely the in-depth flatness, the maximum clinical range $\left(\mathrm{R}_{90}\right)$, and the DFO. All defined SOBPs have an in-depth flatness better than $2 \%$ and a DFO lower than $2 \mathrm{~mm}$. Moreover, we estimated the maximum and minimum dose rates for each option, with a $500 \mathrm{nA}$ cyclotron current, and found out that a treatment session can be delivered at a maximum dose rate of $17 \mathrm{~Gy} / \mathrm{min}$ in single scattering mode. To further increase this dose rate, we studied an alternative design, which combines a thin tantalum scatterer with a cylindrical lead beam stopper, to obtain a flat transverse dose profile. For a given cyclotron current of $500 \mathrm{nA}$, our design can reach a maximum dose rate of $42.4 \mathrm{~Gy} / \mathrm{min}$ (i.e., $0.7 \mathrm{~Gy} / \mathrm{s}$ ), which is three times higher than the maximum value obtained with the single scattering mode (17 Gy/min). Using BDSIM, Monte Carlo simulations were performed to assess the clinical properties of the dose deposition profiles obtained with this design. The results are very similar to those shown by the single scattering mode, namely in terms of DFO, lateral penumbra, and transverse and in-depth flatnesses. Moreover, the beam stopper design

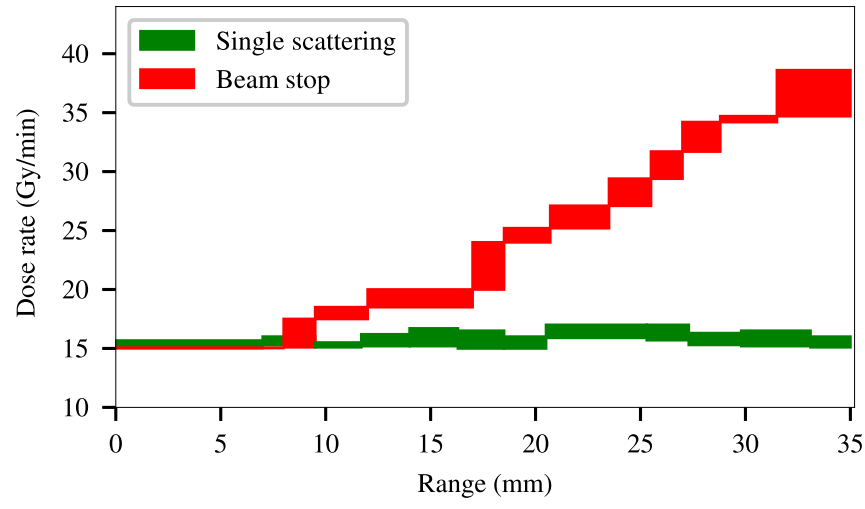

FIG. 20. Schematic representation of the minimum and maximum dose rates of all options defined for the two designs proposed for the eyeline. Each option is represented by a rectangular box, limited by the minimum and maximum ranges (on the left and on the right, respectively) and the minimum and maximum dose rates (on the bottom and on the top, respectively).

allows a slightly smaller dose at the skin (from 30 to $25 \%$ of the maximum delivered dose), which is an additional benefit for the patient regarding the dose given to OARs.

A direct next step for the study presented in this paper is a complete experimental validation, not only of the beamline model, but also of the derived clinical performances, for the two designs studied. Such an experimental validation which is currently ongoing will be of significant benefit, especially in the case of the beam stopper design, knowing that beam stopper devices have rarely been used so far in eye-treatment proton therapy beamlines. Moreover, as the treatment field sizes are particularly small (typically less than $1.5 \mathrm{~cm}$ radius), a sensitivity analysis can be performed to investigate the impact of devices' misalignments on the clinical properties. Simulations could also be performed using more realistic eye and tumor compositions. As presented in [28], the composition used for the simulations can have a significant impact on the predicted clinical range for a given nominal energy of the beam. Moreover, it can affect the flatness and the DFO of the depth dose deposition profiles, less sparing of OARs. Therefore, applying the calibration workflow presented in this paper to more realistic models of eye tumors and OARs can be of great interest, as it will allow a more realistic estimation of the dosimetric properties of the beamline.

\section{ACKNOWLEDGMENTS}

This work has received funding from the Walloon Region (SPW-EER) PIT program under Grant Agreement No. 7289. The authors would like to thank A. Rogé and M. Dechamps for the collection of on-site data and numerous IBA colleagues for all their support given over the years.
[1] Ion Beam Therapy: Fundamentals, Technology, Clinical Applications, edited by U. Linz (Springer, Berlin, 2012).

[2] P. Jovanovic et al., Ocular melanoma: an overview of the current status, Int. J. Clin. Exp. Pathol. 6, 1230 (2013).
[3] K. Tae Wan et al., Clinical outcomes of proton beam therapy for choroidal melanoma at a single institute in Korea, Cancer Res. Treatment: Official J. Korean Cancer Assoc. 50, 335 (2018). 
[4] R. Slopsema et al., Dosimetric properties of a proton beamline dedicated to the treatment of ocular disease, Med. Phys. 41, 011707 (2014).

[5] J. Swakon et al., Facility for proton radiotherapy of eye cancer at IFJ PAN in Krakow, Radiat. Meas. 45, 1469 (2010).

[6] I. K. Daftari et al., New UCSF proton ocular beam facility at the Crocker Nuclear Laboratory Cyclotron (UC Davis), Nucl. Instrum. Methods Phys. Res., Sect. A 380, 597 (1996).

[7] G. Cirrone et al., A 62-MeV proton beam for the treatment of ocular melanoma at Laboratori Nazionali del Sud-INFN, IEEE Trans. Nucl. Sci. 51, 860 (2004).

[8] D. Bonnett, A. Kacperek, M. Sheen, R. Goodall, and T. Saxton, The $62 \mathrm{MeV}$ proton beam for the treatment of ocular melanoma at Clatterbridge, Br. J. Radiol. 66, 907 (1993).

[9] J. Hérault, N. Iborra, B. Serrano, and P. Chauvel, Monte Carlo simulation of a protontherapy platform devoted to ocular melanoma, Med. Phys. 32, 910 (2005).

[10] J. Röhrich, T. Damerow, W. Hahn, U. Müller, U. Reinholz, and A. Denker, A Tandetron as proton injector for the eye tumor therapy in Berlin, Rev. Sci. Instrum. 83, 02 B903 (2012).

[11] E. Blackmore et al., Operation of the TRIUMF proton therapy facility, in Proceedings of the 1997 Particle Accelerator Conference (PAC), Vancouver, BC, Canada (IEEE, 1997), p. 3.

[12] F. Martinetti et al., Monte Carlo modeling of a protontherapy beam line dedicated to ophthalmologic treatments, Nucl. Technol. 168, 721 (2009).

[13] J. Heufelder et al., SU-GG-T-558: OPTIS2-PSI's new ocular proton therapy facility, Med. Phys. 35, 2853 (2008).

[14] E. S. Gragoudas et al., Proton irradiation of small choroidal malignant melanomas, Am. J. Ophthalmol. 83, 665 (1977).

[15] E. S. Gragoudas et al., Proton beam irradiation: An alternative to enucleation for intraocular melanomas, Ophthalmology 87, 571 (1980).

[16] D. Prieels, Calculations and design considerations of the nozzle dedicated to eye irradiation, Tech. rep., Ion Beam Applications, 2006.

[17] L. J. Nevay et al., BDSIM: An accelerator tracking code with particle-matter interactions, Comput. Phys. Commun. 252, 107200 (2020).
[18] S. Agostinelli et al., Geant4-a simulation toolkit, Nucl. Instrum. Methods Phys. Res., Sect. A 506, 250 (2003).

[19] M. Werlen and D. Perret-Gallix, New computing techniques in physics research V, Nucl. Instrum. Methods Phys. Res., Sect. A 389, 1 (1997).

[20] L. Lönnblad, CLHEP - a project for designing a C ++ class library for high energy physics, Comput. Phys. Commun. 84, 307 (1994).

[21] R. Tesse et al., Numerical simulations to evaluate and compare the performances of existing and novel degrader materials for proton therapy, J. Phys.: Conf. Ser. 1067, 092001 (2018).

[22] D. Hall, A. Makarova, H. Paganetti, and B. Gottschalk, Validation of nuclear models in Geant4 using the dose distribution of a $177 \mathrm{MeV}$ proton pencil beam, Phys. Med. Biol. 61, N1 (2016).

[23] R. Tesse et al., Simulations of the activation of a proton therapy facility using a complete beamline model with BDSIM, in Proceedings of the 10th International Particle Accelerator Conference (IPAC), Melbourne, Australia (JaCoW, 2019), pp. 4176-4179.

[24] C. Hernalsteens et al., A novel approach to seamless simulations of compact hadron therapy systems for self-consistent evaluation of dosimetric and radiation protection quantities, Eur. Phys. Lett. 132, 50004 (2020).

[25] S. D. Walker, A. Abramov, L. J. Nevay, W. Shields, and S. T. Boogertt, PYG4OMETRY: A python library for the creation of Monte Carlo radiation transport physical geometries, Comput. Phys. Commun. 272, 108228 (2022).

[26] V. Anferov, Energy degrader optimization for medical beam lines, Nucl. Instrum. Methods Phys. Res., Sect. A 496, 222 (2003).

[27] B. Gottschalk, On the scattering power of radiotherapy protons, Med. Phys. 37, 352 (2010).

[28] S. Keshazare, S. F. Masoudi, and F. S Rasouli, Effects of defining realistic compositions of the ocular melanoma on proton therapy, J. Biomed. Phys. Eng. 4, 141 (2014).

Correction: The omission of institutional names in the first and fourth affiliations in the front matter have been corrected. 